\title{
Parallel T-cell cloning and deep sequencing of human MAIT cells reveal stable oligoclonal TCR $\beta$ repertoire
}

Marco Lepore ${ }^{1}$, Artem Kalinichenko ${ }^{1}$, Alessia Colone ${ }^{2}$, Bhairav Paleja ${ }^{2}$, Amit Singhal ${ }^{2}$, Andreas Tschumi ${ }^{3}$, Bernett Lee ${ }^{2}$, Michael Poidinger ${ }^{2}$, Francesca Zolezzi ${ }^{2}$, Luca Quagliata ${ }^{4}$, Peter Sander ${ }^{3,5}$, Evan Newell ${ }^{2}$, Antonio Bertoletti ${ }^{6}$, Luigi Terracciano ${ }^{4}$, Gennaro De Libero ${ }^{1,2}$ \& Lucia Mori, ${ }^{1,2}$

Mucosal-associated invariant T (MAIT) cells are abundant in humans and recognize conserved bacterial antigens derived from riboflavin precursors, presented by the nonpolymorphic MHC class I-like molecule MR1. Here we show that human MAIT cells are remarkably oligoclonal in both the blood and liver, display high inter-individual homology and exhibit a restricted length CDR3 $\beta$ domain of the TCRV $\beta$ chain. We extend this analysis to a second sub-population of MAIT cells expressing a semi-invariant TCR conserved between individuals. Similar to 'conventional' MAIT cells, these lymphocytes react to riboflavin-synthesizing microbes in an MR1-restricted manner and infiltrate solid tissues. Both MAIT cell types release Th0, Th1 and Th2 cytokines, and SCD4OL in response to bacterial infection, show cytotoxic capacity against infected cells and promote killing of intracellular bacteria, thus suggesting important protective and immunoregulatory functions of these lymphocytes.

\footnotetext{
${ }^{1}$ Experimental Immunology, Department of Biomedicine, University Hospital Basel, 4031 Basel, Switzerland. ${ }^{2}$ SlgN, Singapore Immunology Network, Agency for Science, Technology and Research, Singapore 138648, Singapore. ${ }^{3}$ Institute of Medical Microbiology, University of Zurich, 8006 Zurich, Switzerland. ${ }^{4}$ Institute of Pathology, University Hospital Basel, 4031 Basel, Switzerland. ${ }^{5}$ National Centre for Mycobacteria, Gloriastrasse 30/32, 8006 Zurich, Switzerland. ${ }^{6}$ Emerging Infectious Diseases, Duke-NUS Graduate Medical School, Singapore 169857, Singapore. Correspondence and requests for materials should be addressed to G.D.L. (email: gennaro.delibero@unibas.ch) or to L.M. (email: lucia_mori@immunol.a-star.edu.sg).
} 
M ucosal-associated invariant T (MAIT) cells represent up to $10 \%$ of circulating $\mathrm{T}$ cells in humans and are abundant in the gastrointestinal mucosa and liver ${ }^{1-4}$.

Although MAIT cells are known to respond to Gram-positive bacteria, Gram-negative bacteria and yeasts, ${ }^{5,6}$, and have antimicrobial potential ${ }^{7-10}$, there is no consensus on their physiological role. Antigen recognition by MAIT cells is mediated by an $\alpha \beta$ T-cell receptor (TCR), in which the TCRV $\alpha$ chain comprises an almost-invariant V $\alpha 7.2-\mathrm{J} \alpha 33$ (TRAV1-2TRAJ33) rearrangement paired with a limited number of TCRV $\beta$ chains ${ }^{1}$. These limited $\mathrm{V} \alpha-\mathrm{V} \beta$ heterodimers are analogous to those of invariant natural killer T cells (iNKT) ${ }^{11}$, and the two cell populations also share expression of NK-related molecules such as CD161 in humans $s^{2,12}$ and similarly acquire an effector/ memory phenotype after birth ${ }^{2,13}$. In contrast to NKT cells, MAIT cells appear in the periphery following bacterial colonization $^{14}$, suggesting that commensal bacteria-derived signals and/or antigens are important in sustaining their expansion and survival. MAIT cells recognize small microbial metabolites produced in the riboflavin pathway ${ }^{15}$, when presented as stimulatory complexes with the non-polymorphic major histocompatibility complex (MHC) class I-like related molecule 1 (MR1) ${ }^{16-18}$. Whether riboflavin-related metabolites are the only antigens stimulating MAIT cells, and how these antigens affect MAIT cell expansion and homeostasis remains to be elucidated.

Another unresolved issue is how the TCR repertoire of the invariant T-cell populations is generated; whether it is predominantly antigen-driven or is dependent on random but convergent TCR gene rearrangement mechanisms. The almostinvariant TCR $\alpha$ chain of MAIT cells is evolutionarily conserved in mammals ${ }^{14,19}$ and results from a single $\mathrm{V} \alpha$-J $\alpha$ rearrangement with a defined $\operatorname{CDR} 3 \alpha$ length. In humans, some variation in the composition of the junction exists, suggesting a selective pressure operating at the protein level ${ }^{14}$. Nevertheless, a recent computer simulation of gene recombination events proposed that the invariant TCR $\alpha$ chains of MAIT and NKT cells can be generated by a recombination process involving various convergent mechanisms ${ }^{20}$.

Studies comparing a limited number of TCR sequences have suggested that the TCR $\beta$ repertoire of MAIT cells might be biased toward V $\beta 2$ (TRBV20) and V $\beta 13$ (TRBV6), but failed to detect any preferential use of particular $\mathrm{J} \beta$ genes or identify the prevalence of defined CDR3 $\beta$ clonotypes ${ }^{1,21}$. However, the investigation of small numbers of MAIT TCR gene sequences precludes a thorough examination of the impact of antigen stimulation on shaping the TCR repertoire and of the TCR $\beta$ chain role in antigen recognition.

MAIT cells are abundant in tissues exposed to microbial antigens, including the gut, liver and infected lung ${ }^{2,5,22}$. Their parallel abundance in the blood also suggests that they may recirculate between these organs. The oligoclonality of tissue-resident $\mathrm{T}$ lymphocytes was described long before the discovery of MAIT cells ${ }^{23,24}$, but it is unknown which of these oligoclonal $\mathrm{T}$ cells recognize microbial antigens presented by MR1.

Here we define the TCR $\beta$ repertoire of MAIT cells in healthy humans by single cell cloning and mRNA deep sequencing experiments, and we show that a small number of clonotypes accounts for the majority of MAIT cells in both blood and liver. Furthermore, we describe a second population of MAIT cells expressing a different invariant $\mathrm{TCR} \alpha$ chain. We also investigate the tissue distribution and effector functions of this novel subset in comparison with 'conventional' MAIT cells.

\section{Results}

Isolation and characterization of human MAIT cell clones. Human MAIT cells are CD4-negative and express high levels of the NK-related marker CD161 (ref. 2). To establish MAIT cell clones, $\mathrm{CD} 4{ }^{-} \mathrm{CD} 161^{\text {high }} \mathrm{T}$ cells were sorted from peripheral blood of a healthy donor (A) and cloned by limiting dilution. As iNKT and TCR $\gamma \delta$ T cells also express CD161 and may lack CD4, they were excluded from sorting using antibodies specific for the TCR V $\alpha 24$ (ref. 25) and V $\delta 2$ chains ${ }^{26}$ (Supplementary Fig. 1a). In preliminary experiments, a significant proportion of these $\mathrm{CD} 4^{-} \mathrm{V} \delta 2^{-} \mathrm{V} \alpha 24^{-} \mathrm{CD} 161^{+} \mathrm{T}$ cells upregulated surface expression of CD69 after co-culture with autologous monocytes infected with Escherichia coli (Supplementary Fig. 1b). When the individual T-cell clones were tested by PCR, 15 clones expressed the MAIT TCR V $\alpha 7.2-\mathrm{J} \alpha 33$ rearrangement. Each T-cell clone was further characterized for the TCRV $\beta$ chain expressed and for surface expression of CD161, CD4 and CD8 by flow cytometry.

Thirteen clones possessed V $\beta 2$ ( $B V 2 S 1$ according to ref. 27 or TRBV20-1 according to ref. 28) or V $\beta 13$ (BV13S3 and BV13S5 or TRBV6-1 and TRBV6-4) chains, both reported to be preferentially

Table 1 | Phenotype and amino acid TCR $\beta$ sequences of $\mathrm{V} \alpha 7.2-\mathrm{J} \alpha 33$ T-cell clones from donor $A$.

\begin{tabular}{|c|c|c|c|c|c|c|}
\hline $\operatorname{mrc} 26$ & - & + & + & BV6-1, BD2, BJ2-7 & ASRLMSGSSYEQY & 13 \\
\hline $\operatorname{mrc35}$ & - & + & + & BV6-1, BD2, BJ2-7 & ASRLMSGSSYEQY & 13 \\
\hline $\operatorname{mrc} 36$ & - & + & + & BV6-1, BD2, BJ2-7 & ASRLMSGSSYEQY & 13 \\
\hline $\operatorname{mrc65}$ & - & + & + & BV6-1, BD2, BJ2-7 & ASRLMSGSSYEQY & 13 \\
\hline mra8 & - & + & + & BV20-1, BD1, BJ1-1 & SARDRRETEAF & 11 \\
\hline mrb21 & - & + & low & BV20-1, BD1, BJ1-1 & SARDRRETEAF & 11 \\
\hline $\operatorname{mrc66}$ & - & + & + & BV20-1, BD1, BJ1-1 & SARDRRETEAF & 11 \\
\hline mra9 & - & + & + & BV20-1, BD2, BJ2-1 & SARGDREAYNEQF & 13 \\
\hline mra13 & - & + & low & BV20-1, BD2, BJ2-1 & SARGDREAYNEQF & 13 \\
\hline mrb29 & - & + & low & BV20-1, BD2, BJ2-1 & SARGIDRVTNEQF & 13 \\
\hline mrb24 & - & - & + & BV4-3, BD2, BJ2-5 & ASSQERGSQETQY & 13 \\
\hline
\end{tabular}


expressed in MAIT cells ${ }^{1}$ (Table 1). Two clones instead expressed the V $\beta 7.2$ (BV7S2 or TRBV4-3) and V $\beta 16$ (BV16S1 or TRBV14) chains, respectively (Table 1$)$. The CD8 molecule was present in 13 clones, whereas one clone was $\mathrm{CD}^{-}{ }^{-} \mathrm{CD}^{-}$(Table 1). All $\mathrm{T}$-cell clones were $\mathrm{CD} 4^{-}$and $\mathrm{CD} 161^{+}$(Table 1), indicating that no bias was introduced by the sorting and cloning strategies. As MAIT cells recognize antigen-presenting cells (APC) infected with Gram-positive or Gram-negative bacteria ${ }^{5,6}$, three different bacterial strains were used to infect APC and study stimulation of the selected T-cell clones. Monocyte-derived dendritic cells or THP-1 myelomonocytic cells, which express surface MR1 (Supplementary Fig. 1c) were infected with E. coli (Gramnegative), Staphylococcus aureus (Gram-positive) and Mycobacterium smegmatis, and co-cultured with T-cell clones. Each tested clone responded to all the infected cells, regardless of APC type used and of the $V \beta$ chain expressed by the clone (Table 1 and Supplementary Fig. 1d). Addition of anti-MR1 monoclonal antibodies (mAbs) inhibited T-cell responses (Supplementary Fig. 1d), but did not affect activation of MHC class II-restricted Mycobacterium tuberculosis-specific or CD1brestricted $M$. tuberculosis-reactive $\mathrm{T}$ cells, used as controls (Supplementary Fig. 1e). Taken together, these data indicated that established V $\alpha 7.2-\mathrm{J} \alpha 33 \mathrm{~T}$-cell clones are bona fide MAIT cells, as they express the prototypic markers of this population, their responses are restricted by MR1 and they recognize APC infected with riboflavin-producing bacteria.

MAIT cells possess a limited TCR $\beta$ repertoire. The analysis of TCRV $\beta$ sequences of 15 MAIT cell clones revealed that they represented only 7 different CDR3 $\beta$ amino acid clonotypes; moreover, three of these clonotypes together accounted for 11 clones (73\%), being expressed in 5, 4 and 2 clones, respectively (Table 1). As the T-cell clones were generated with neither previous stimulation nor in vitro expansion, it appeared that the circulating MAIT cell population might in fact originate from a small number of in vivo clonally expanded cells. To test this hypothesis, we investigated the TCR $\beta$ repertoire composition of the blood MAIT cells of healthy donors using RNA deep sequencing. MAIT lymphocytes were isolated by polychromatic flow cytometric cell sorting according to co-expression of TCRV 27.2 and CD161 $1^{\text {high }}$ (Supplementary Fig. 2), and TCR $\beta$ sequences were obtained from the sorted population. Non-MAIT cell subsets were defined by a combination of the same markers (V $\alpha 7.2^{+} \mathrm{CD}_{161}{ }^{-}, \mathrm{V} \alpha 7.2^{-} \mathrm{CD} 161^{+}$and $\mathrm{V} \alpha 7.2^{-}$ $\left.\mathrm{CD}_{161^{-}}\right)$and were simultaneously sorted and analysed as controls (Supplementary Fig. 2). These studies were performed in peripheral blood mononuclear cells (PBMC) from six healthy donors $(1,2 \mathrm{~B}, 3,4,5$ and 6$)$ and in two samples of liver-associated mononuclear cells. The total sequences analysed were between 103,704 and 17,023,718 per donor and sorted T-cell population, and they showed a range of 2,258-438,029 unique sequences in all four T-cell subsets (Supplementary Table 1).

A strong bias in TCRV $\beta$ usage was observed in MAIT cells, as previously reported ${ }^{1}$. V $\beta 13$ (TRBV6) and V $\beta 2$ (TRBV20) families together accounted for $32-85 \%$ of the total MAIT cell repertoire, with some inter-donor variability (Fig. 1a, upper panel). Heterogeneous $\mathrm{V} \beta$ usage was evident in $\mathrm{V} \alpha 7.2^{+} \mathrm{CD} 161^{-}$ control $\mathrm{T}$ cells, thus excluding the possibility that the observed MAIT cell V $\beta$ bias was associated with the presence of this TCRV $\alpha$ chain (Fig. 1a, lower panel). Although a predominant expression of individual J $\beta$ genes was not detected in MAIT cells compared with control $\mathrm{T}$ lymphocytes, significant differences were observed in the distribution of unique CDR3 $\beta$ amino acid clonotypes. In the MAIT cell population, $5 \%$ of the unique clonotypes with the largest size accounted for $77-82 \%$ of the total
TCR $\beta$ repertoire (Fig. $1 \mathrm{~b}, \mathrm{c}$ ). By contrast, the same $5 \%$ covered only $33-64 \%, 36-66 \%$ and $31-51 \%$ of the repertoire in $\mathrm{V} \alpha 7.2^{+} \mathrm{CD} 161^{-}, \mathrm{V} \alpha 7.2^{-} \mathrm{CD} 161^{+}$and $\mathrm{V} \alpha 7.2^{-} \mathrm{CD} 161^{-}$ control $\mathrm{T}$ cells, respectively (Fig. $1 \mathrm{~b}, \mathrm{c})$. These data indicated a marked oligoclonality of CDR $3 \beta$ amino acid clonotypes in MAIT cells, with relatively few clones accounting for the majority of the overall repertoire. Indeed, an average of 250 unique sequences were sufficient to represent $50 \%$ of the total MAIT cell repertoire, while 3,000-30,000 sequences on average covered the same proportion of the TCR repertoire in non-MAIT cell populations (Fig. 1d). Deep-sequence analysis performed on sorted V $\alpha 7.2^{+}$CD $161^{\text {high }}$ liver-associated MAIT lymphocytes, which can account for up to half the resident T-cell population of this organ $^{2,22}$, revealed a distribution of CDR3 $\beta$ clonotypes comparable to that observed in peripheral blood (Supplementary Fig. 3). In the two liver samples, 5\% of the largest CDR3 $\beta$ clonotypes represented 84.3 and $81.6 \%$ of the total repertoire, respectively, thus suggesting that oligoclonality is a shared feature of both the liver-resident and circulating pool of MAIT cells.

To confirm the above observations, from the same V $\alpha 7.2^{+}$ $\mathrm{CD} 161^{\text {high }}$ cells sorted from donor $2 \mathrm{~B}$ and analysed in parallel for TCR $\beta$ gene deep sequencing, a second, independent, panel of MAIT cell clones was generated. Within this panel, TCR $\beta$ sequence screening of $14 \mathrm{~V} \alpha \mathbf{7}$.2-J $\alpha 33$-positive T-cell clones revealed only 8 different CDR3 $\beta$ clonotypes, 2 of which accounted for more than half of the cell clones (Table 2). Deep sequencing showed that these eight sequences were highly frequent in the $\mathrm{V} \alpha 7.2^{+} \mathrm{CD} 161^{\text {high }}$ cell subset from which the clones were derived, together representing $>10 \%$ of the total TCR $\beta$ repertoire of MAIT cells from that donor, although being almost undetectable in the other control T-cell subsets (Supplementary Table 2). Taken together, these findings provided a precise enumeration of the size of MAIT cell clonotypes in healthy donors and unequivocally demonstrated the oligoclonality of the MAIT population in the blood and liver.

MAIT cell CDR3 $\beta$ clonotypes are stable in blood over time. We next asked whether the oligoclonality observed in circulating MAIT cells is a stable or dynamic phenomenon. To address this issue, $\mathrm{V} \alpha 7.2^{+} \mathrm{CD} 161^{\text {high }}$ cells were sorted from peripheral blood of three donors $(3,4$ and 5$)$ at two different time points separated by a 5-month interval, before parallel analysis of MAIT cell CDR3 $\beta$ clonotypes by mRNA deep sequencing (Supplementary Table 1$)$. In every individual, the observed CDR3 $\beta$ clonotypes were substantially unchanged, exhibiting comparable distribution and size within the total repertoire (Fig. 1e and Supplementary Fig. 4). Importantly, the unique amino acid sequences common to the two time points represented between 99.6 and $99.9 \%$ of the total repertoire size. Few clonotypes were detected at one but not the other time point. These data suggested that within the investigated timeframe the majority of the circulating MAIT cell repertoire is stable in human peripheral blood.

Inter-individual sharing of MAIT TCR $\beta$ clonotypes. CDR3 $\beta$ clonotypes can be shared between different individuals ${ }^{29}$, implying a certain degree of TCR repertoire overlap in peripheral blood, although whether this similarly applies to the MAIT population is unknown. As MAIT cells uniquely recognize a common microbial antigen ${ }^{15}$ and are oligoclonal, we hypothesized that they might also display a high degree of TCR repertoire overlap between different individuals. We therefore evaluated the extent of TCR $\beta$ repertoire sharing within the peripheral blood MAIT cell population between paired donors with respect to unique amino acid sequences and also to the 

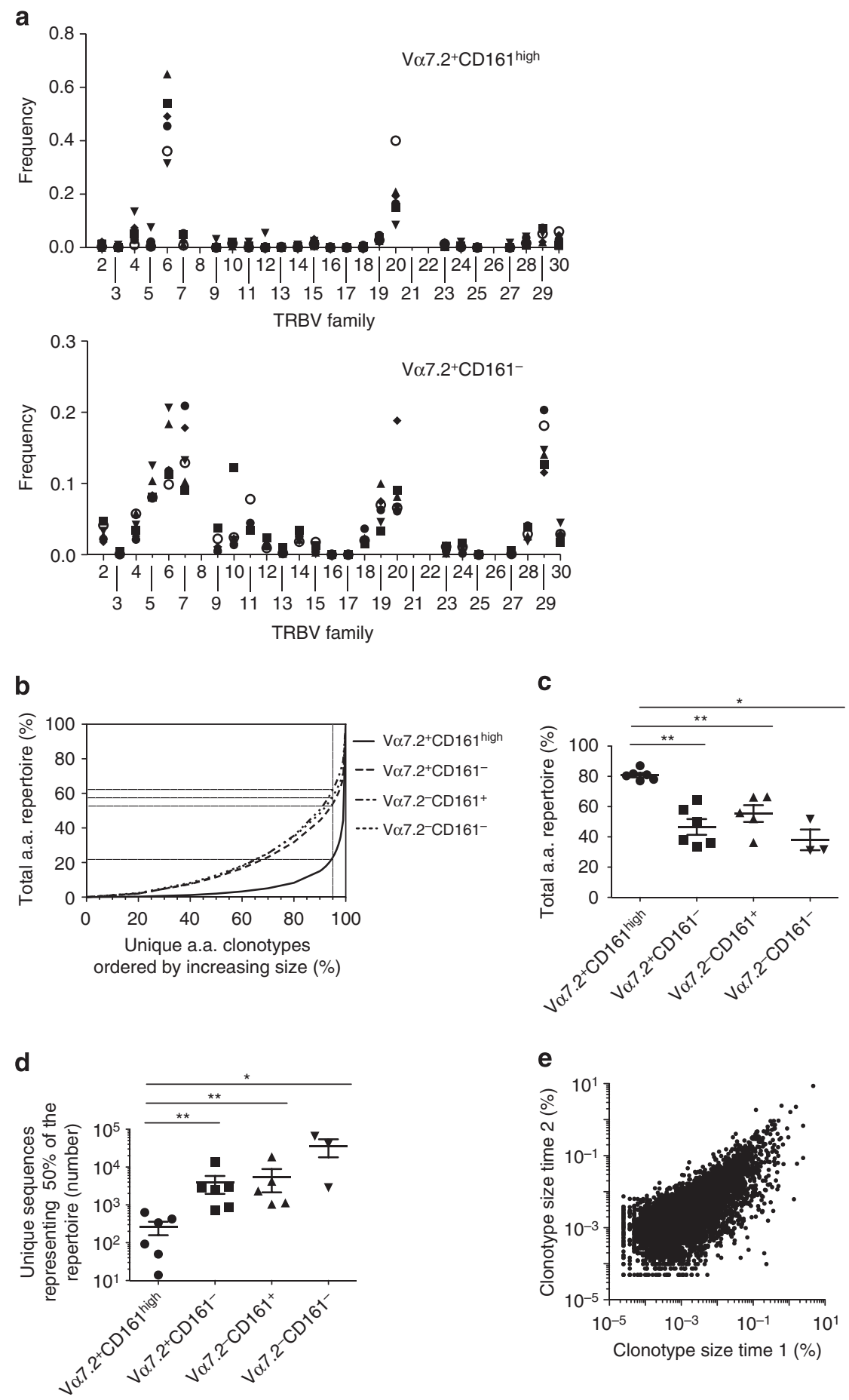

Figure 1 | MAIT cells are oligoclonal in human peripheral blood. (a) V $\beta$ usage of sorted peripheral blood $V \alpha 7.2+C D 161^{\text {high }}$ MAIT cells (upper panel) and $\mathrm{V} \alpha 7.2^{+} \mathrm{CD} 161^{-}$control T cells (lower panel). IMGT nomenclature is used. Each point represents data from an individual healthy donor. (b) Distribution of clonotype sizes in $\mathrm{V} \alpha 7.2^{+} \mathrm{CD} 161^{\text {high }}$ MAIT cells, and $\mathrm{V} \alpha 7.2^{+} \mathrm{CD} 161^{-}, \mathrm{V} \alpha 7.2^{-} \mathrm{CD} 161^{-}$and $\mathrm{V} \alpha 7.2^{-} \mathrm{CD} 161^{+}$control T cells from a representative donor. Data are plotted as percentage of total TCR $\beta$ amino acid (a.a.) repertoire versus the percentage of unique TCR $\beta$ amino-acid repertoire ordered by increasing size. The $y$-value at which each curve intersects the dashed line $(x=95 \%)$ represents the percent value of the total repertoire covered by $95 \%$ of unique clonotypes for each T-cell population. The graph shows that $5 \%$ of the most abundant clonotypes, evaluated as amino-acid, contribute $77 \%$ of the total TCR $\beta$ repertoire of $\mathrm{V} \alpha 7.2^{+} \mathrm{CD} 161^{\text {high }}$ MAIT cells and only $38 \%, 56 \%$ and $52 \%$ of that of $\mathrm{V} \alpha 7.2^{+} \mathrm{CD}_{161}{ }^{-}, \mathrm{V} \alpha 7.2^{-} \mathrm{CD} 161^{+}$and Va7.2- CD161- control T-cell populations, respectively. (c) Percentage of total TCR $\beta$ amino-acid repertoire represented by $5 \%$ of the largest unique amino-acid clonotypes for the indicated T-cell populations in all donors analysed. For every T-cell subset, each point represents a different donor. Bars indicate mean \pm s.e.m. $\left({ }^{\star} P \leq 0.05,{ }^{\star}{ }^{\star} P \leq 0.01\right.$, determined by Mann-Whitney $U$-test). (d) Number of unique amino-acid sequences contributing the $50 \%$ of the total TCR $\beta$ amino-acid repertoire of the indicated T-cell populations. For every T-cell subset, each point represents data from a different donor. Bars indicate mean \pm s.e.m. ( ${ }^{\star} P \leq 0.05,{ }^{\star \star} P \leq 0.01$, determined by Mann-Whitney $U$-test). (e) Size of all individual TCR $\beta$ amino-acid clonotypes in $\mathrm{V} \alpha 7.2^{+} \mathrm{CD} 161^{\text {high }}$ MAIT cells of a representative donor (3) analysed at two different time points with a 5-month interval. 
Table 2 | Phenotype and amino acid TCRß sequences of V $\alpha 7.2-J \alpha 33$ T-cell clones from donor $2 B$.

\begin{tabular}{|c|c|c|c|c|c|c|}
\hline \multirow[t]{2}{*}{ T-cell clone } & \multirow[t]{2}{*}{ CD4 } & \multirow[t]{2}{*}{ CD8 } & \multirow[t]{2}{*}{ CD161 } & \multicolumn{3}{|c|}{ TCR $\beta$} \\
\hline & & & & V-D-J & CDR3 & CDR3 length \\
\hline 20.7B6 & - & nd & + & BV6-5, BD2, BJ2-5 & ASSPSGGGAQETQY & 14 \\
\hline $20.7 B 66$ & - & nd & + & BV6-1, BD1, BJ2-7 & ASSVGQENYEQY & 12 \\
\hline $20.7 A 24$ & - & nd & + & BV6-6, BD2, BJ2-3 & ASSNRVTSTDTQY & 13 \\
\hline $20.7 B 60$ & - & nd & + & BV20-1, BD2, BJ2-2 & SATGTGDTGELF & 12 \\
\hline 20.7B25 & - & nd & + & BV20-1, D1, J1-4 & SAPWAGVNEKLF & 12 \\
\hline 20.7B52 & - & nd & + & BV20-1, D1, J1-4 & SAPWAGVNEKLF & 12 \\
\hline $20.7 B 69$ & - & nd & + & BV20-1, D1, J1-4 & SAPWAGVNEKLF & 12 \\
\hline $20.7 B 72$ & - & nd & + & BV20-1, D1, J1-4 & SAPWAGVNEKLF & 12 \\
\hline $20.7 A 26$ & - & nd & + & BV4-2/3, DB, BJ2-3 & ASSQEGQGAPTDTQY & 15 \\
\hline 20.7B71 & - & nd & + & BV24/OR9-2, BD2, BJ2-7 & ATSREGRASNEQY & 13 \\
\hline
\end{tabular}

cumulative repertoire size. In MAIT cells, $2 \pm 0.39 \%$ of the unique CDR3 $\beta$ amino acid clonotypes present in one individual were also present in a second individual (Fig. 2a,c, upper panel). Furthermore, $7.3 \pm 2.7 \%$ of the total TCR $\beta$ repertoire overlapped in size between paired donors, with individuals sharing up to $80 \%$ (Fig. 2c, lower panel). When the same analysis was performed in non-MAIT $\mathrm{V} \alpha 7.2^{+} \mathrm{CD} 161^{-}$and $\mathrm{V} \alpha 7.2^{-} \mathrm{CD} 161^{-}$cell populations, the proportion of unique CDR3 $\beta$ amino acid clonotypes and total size of shared TCR $\beta$ repertoire by paired donors dropped significantly to $1 \pm 0.19$ and $0.65 \pm 0.29 \%$, respectively, for unique clonotypes (Fig. 2b,c, upper panel) and $1.6 \pm 0.4$ and $0.52 \pm 0.12 \%$, respectively, for repertoire size (Fig. 2c, lower panel). Thus, the degree of TCR $\beta$ repertoire sharing between different individuals is substantially higher in MAIT than in non-MAIT cell populations.

We also evaluated the proportion of CDR3 $\beta$ clonotypes common to all the donors in MAIT and non-MAIT cell pools. Although in the latter group no public clonotypes were observed, in MAIT cells one identical CDR3 $\beta$ clonotype was detected in all analysed individuals, including the samples from the liver, further supporting the conclusion that some MAIT cell TCR $\beta$ sequences are commonly shared between different donors.

CDR3 $\beta$ length distribution differs in MAIT and non-MAIT cells. A recent study suggested that the TCR $\beta$ chain might be less important for MAIT cell stimulation than was previously thought ${ }^{30}$. To investigate whether the TCRV $\beta$ chains of MAIT cells have unique features, we compared the CDR3 $\beta$ length in MAIT and non-MAIT cells at amino acid level. In MAIT cells, a predominance of four CDR3 $\beta$ lengths was observed. In all donors, the dominant length was 13 amino acids, followed by 12 , 11 and 14 with inter-individual heterogeneity (Fig. 2d). No significant bias was observed in control T-cell pools from the same individuals (Fig. 2d), indicating that the abundance of clonotypes with a restricted CDR3 $\beta$ length is a specific feature of MAIT lymphocytes. These observations were further supported by the analysis of the CDR3 $\beta$ amino acid clonotypes of all generated MAIT cell clones, which showed a size ranging between 11 and 14 amino acids, with only one exception (Tables 1 and 2).

The co-crystal of the TCR of MAIT cells and MR1 showed that CDR3 $\beta$ residues form van der Waals interactions with the MR1 $\alpha 1$ helix, thereby contributing to the overall interaction between the two proteins ${ }^{31,32}$. The preferential length of MAIT CDR3 $\beta$ sequences might contribute to optimal recognition of MR1antigen complexes.

Isolation of a second semi-invariant MAIT cell population. From sorted $\mathrm{V} \alpha 7.2^{+} \mathrm{CD} 161^{\text {high }}$ cells of donor $2 \mathrm{~B}$, we also isolated six T-cell clones, which were positively labelled by anti$\mathrm{V} \alpha 7.2$ and anti-CD161 mAbs but did not express the V $\alpha 7.2-\mathrm{J} \alpha 33$ transcript. TCR $\alpha$ sequence analysis showed that they all transcribed a functional V $\alpha 7.2-\mathrm{J} \alpha 12$ (TRAV1-2-TRAJ12) rearrangement with identical germline-encoded CDR3 sequence (Supplementary Table 3). Importantly, the same TCR $\alpha$ sequence was found also in three T-cell clones derived from sorted $\mathrm{V} \alpha 7.2^{+} \mathrm{CD} 161^{\text {high }}$ MAIT cells from a third donor (C) (Supplementary Table 3). We were intrigued by the isolation of such T-cell clones from two different donors; therefore, we investigated the reactivity and the restriction of those $\mathrm{T}$ cells. All T-cell clones tested were efficiently stimulated by $E$. coli-infected THP-1 cells and were restricted by MR1, as anti-MR1-mAbs inhibited their activation (Fig. 3a). They were also stimulated by infected Daudi cells, which are naturally $\beta 2$-microglobulin deficient, following transfection with an MR1- $\beta 2 \mathrm{~m}$ fusion gene construct (Supplementary Fig. 5). Infected wild-type Daudi cells lacking MR1 (Supplementary Fig. 5a) did not stimulate Va7.2$\mathrm{J} \alpha 12$ or V $\alpha 7.2-\mathrm{J} \alpha 33 \mathrm{~T}$ clones (Supplementary Fig. 5b). All T-cell clones expressing the V $\alpha 7.2-\mathrm{J} \alpha 12$ invariant rearrangement also reacted to APC infected with $S$. aureus and M. smegmatis, which both produce riboflavin precursors ${ }^{15}$, but not to APC infected with Listeria monocytogenes, which does not synthesize riboflavin (Fig. 3b). In light of the MR1 restriction and the antigenresponsiveness pattern, we assigned the identified $\mathrm{T}$-cell clones to a new sub-population of semi-invariant MAIT cells.

To address whether this novel subset was shared among different individuals, we exploited deep sequencing of total mRNAs to measure the frequency of $\mathrm{V} \alpha 7.2-\mathrm{J} \alpha 12$ transcripts within circulating lymphocytes across 17 normal individuals. Total extracted mRNA was used without amplification with TCRspecific primers. In-frame sequences spanning the V $\alpha 7.2-J \alpha$ region were selected. As these sequences were randomly obtained without previous PCR amplification of mRNA with TCR-specific primers and because of the short reads provided by this method, the number of in-frame sequences recorded was low. Nevertheless, they were deemed sufficient to provide unbiased qualitative and quantitative information about the relative 
a

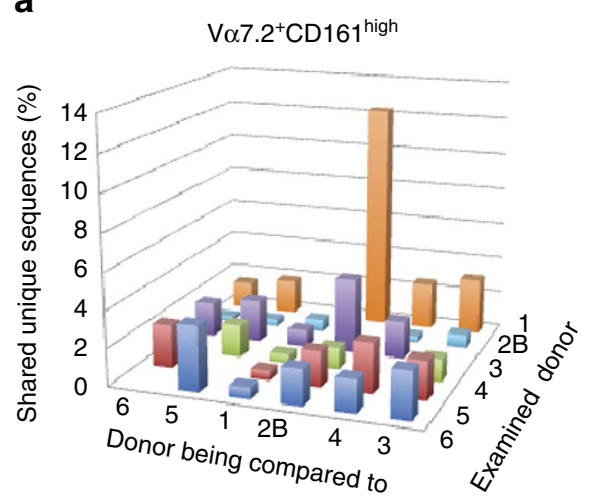

b

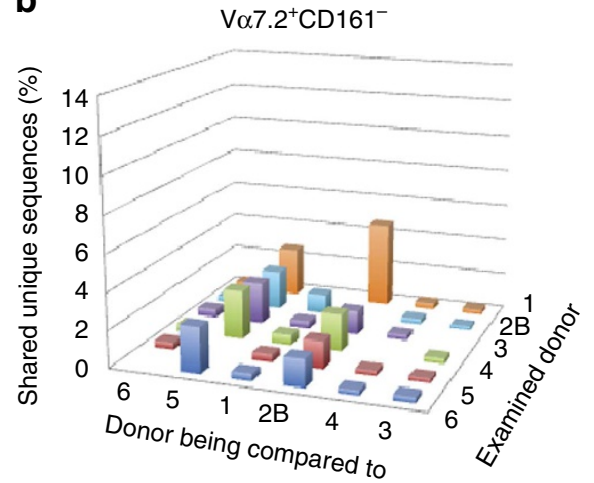

C
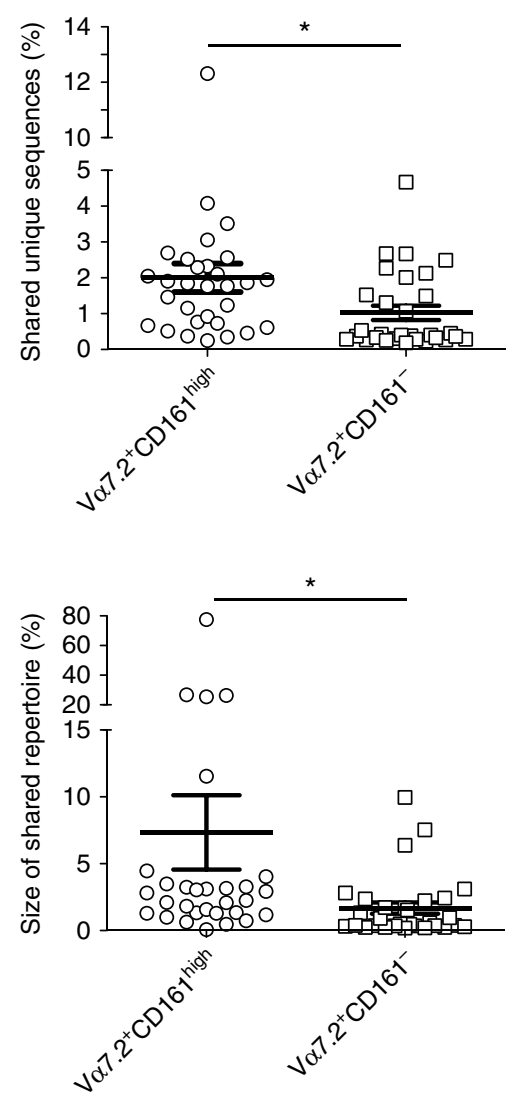

d

- $\mathrm{V} \alpha 7.2^{+} \mathrm{CD} 161^{\mathrm{high}}$

$\square \mathrm{V} \alpha 7.2^{+} \mathrm{CD} 161^{-}$

$\square \mathrm{V} \alpha 7.2^{-} \mathrm{CD} 161^{-}$
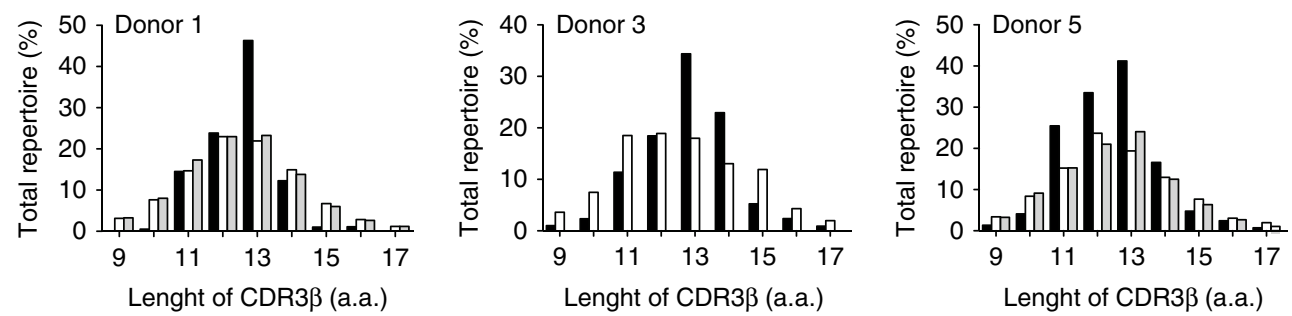

Figure 2 | MAIT cells exhibit a high degree of inter-individual TCR $\beta$ amino-acid sequence sharing and a bias in CDR3 $\beta$ length distribution. Percentage of unique amino-acid sequences shared between donor pairs in (a) $\mathrm{V} \alpha 7.2^{+} \mathrm{CD} 161^{\text {high }}$ MAIT cells and (b) $\mathrm{V} \alpha 7.2^{+} \mathrm{CD} 161^{-}$control T cells. (c) Distribution of TCR $\beta$ repertoire overlapping between paired donors in $\mathrm{V} \alpha 7.2^{+} \mathrm{CD} 161^{\text {high }}$ MAIT cells and $\mathrm{V} \alpha 7.2^{+} \mathrm{CD} 161^{-}$T cells in terms of unique amino-acid clonotypes (upper panel) and cumulative repertoire size (lower panel). Each point represents the percentage of unique amino-acid sequences (upper panel) and total TCR $\beta$ repertoire (lower panel) shared between two donors. Bars indicate mean \pm s.e.m. $\left({ }^{\star} P \leq 0.05\right.$, determined by two-tailed unpaired Student's $t$-test with Welch's correction). (d) Distribution of CDR3 $\beta$ lengths for the total TCR $\beta$ amino-acid (a.a.) repertoire (accounting for clonotype size) in the indicated sorted T-cell populations of three representative donors (1, 3 and 5).

abundance of each different $\mathrm{V} \alpha 7.2-\mathrm{J} \alpha$ rearrangement in individual donors. The V $\alpha 7.2-\mathrm{J} \alpha 33$ rearrangements were the most frequent (total of 68 sequences found in 15 out of 17 donors) (Fig. $3 \mathrm{c}$ and Table 3 ) with the predominant amino acid CAVXDSNYQL motif at the V-J junction as previously reported $^{1}$ and as found in our V $\alpha 7.2$-J $\alpha 33$ MAIT cell clones (Supplementary Table 3). The second most frequent rearrangement was the $\mathrm{V} \alpha 7.2-\mathrm{J} \alpha 12$ (total of 37 sequences detected in 8 out of 17 donors) (Fig. $3 \mathrm{c}$ and Table 3 ). All but 1 of the $37 \mathrm{~V} \alpha$ chains possessed an amino acid sequence at $\mathrm{V}-\mathrm{J}$ junctional regions with the CAVXDSSYKL motif (Table 3) in agreement with that observed also in the V $\alpha 7.2-\mathrm{J} \alpha 12 \mathrm{~T}$-cell clones (Supplementary Table 3 ). In some instances, $N$ nucleotides were present together with variable trimming of the $\mathrm{V} \alpha$ and $\mathrm{J} \alpha$ gene segments, resembling the previously reported joining regions in human V $\alpha 7.2$-J $\alpha 33$ MAIT cells ${ }^{1}$. A third frequent population was characterized by the $\mathrm{V} \alpha 7.2-\mathrm{J} \alpha 20$ rearrangement (12 sequences detected), and in all these $\mathrm{V}-\mathrm{J}$ junctions an extensive remodelling of germline nucleotides was present, resulting in major junction variability (Table 3 ). The rearrangements involving other J $\alpha$ genes were much less frequently represented (Table 3). As a control, the sequences containing the $V \alpha 24$ gene revealed an expectedly high frequency of the invariant $V \propto 24-\mathrm{J} \alpha 18$ rearrangement canonical for iNKT cells, thus illustrating the appropriateness of this type of analysis. Therefore, among the T cells expressing the V $\alpha 7.2 \mathrm{TCR} \alpha$ chain, together with V $\alpha 7.2-\mathrm{J} \alpha 33$, other populations of $\mathrm{T}$ cells 
expressing a $\mathrm{V} \alpha 7.2-\mathrm{J} \alpha 12$ or a $\mathrm{V} \alpha 7.2-\mathrm{J} \alpha 20$ rearrangement are significantly represented in the circulating blood of healthy individuals.

Altogether, these data suggested that $\mathrm{T}$ cells with invariant V $\alpha 7.2-J \alpha 12$ TCR chain represent a second population of human MAIT cells with an apparently similar antigen-responsiveness profile to that described for V $\alpha 7.2-J \alpha 33$-expressing cells ${ }^{5,6,15,21}$.

V $\alpha 7.2-\mathrm{J} \alpha 12$ MAIT cells are oligoclonal. Analysis of the TCR $\beta$ amino acid sequences of the six $\mathrm{V} \alpha 7.2-\mathrm{J} \alpha 12$ clones revealed that they all expressed gene segments of the V $\beta 13$ (TRBV6) family (Table 4). Furthermore, they were represented by only two different CDR3 $\beta$ clonotypes, which were expressed in four and two clones, respectively (Table 4 ). These observations suggested that $\mathrm{V} \alpha 7.2-\mathrm{J} \alpha 12$ MAIT cells might be biased in their V $\beta$ usage and characterized by a restricted TCR $\beta$ repertoire, as for $\mathrm{V} \alpha 7.2$-J $\alpha 33$ MAIT lymphocytes. To investigate this hypothesis, we determined the frequency of these two CDR3 $\beta$ clonotypes in the cell population from which the clones were generated, taking advantage of the mRNA deep sequencing performed on the same population. Both clonotypes were highly frequent in the $\mathrm{V} \alpha 7.2^{+} \mathrm{CD} 161^{\text {high }}$ subset, representing 12 and $0.28 \%$, respectively, of the total TCR $\beta$ repertoire (Supplementary Table 2). In contrast, these same clonotypes were very rare in the three control non-MAIT populations from the same individual (Supplementary Table 2). These data suggested that the circulating V $\alpha 7.2-\mathrm{J} \alpha 12^{+}$MAIT cell population is not a rare population in human blood, and moreover is comprises a relatively small number of clonotypes.

Effector functions of $\mathrm{V} \alpha 7.2-\mathrm{J} \alpha 12^{+}$and $\mathrm{V} \alpha 7.2-\mathrm{J} \alpha 33^{+}$MAIT cells. To compare the effector functions of $\mathrm{V} \alpha 7.2-\mathrm{J} \alpha 12$ and V $\alpha 7.2-J \alpha 33$ MAIT cell populations, their ability to kill bacterialinfected cells was first assessed. Both MAIT cell types efficiently induced apoptosis in E. coli-infected THP-1 cells (Fig. 3d). Importantly, they also induced killing of intracellular mycobacteria and released interferon- $\gamma$ (IFN- $\gamma$ ) and granulocyte macrophage colony-stimulating factor (Fig. 3f), illustrating their capacity for a protective function. This observation was supported by the detection of surface CD107a on T cells, indicating the release of cytotoxic granules, and by the upregulation of intracellular granzyme B expression (Fig. 3e). Downregulation of surface CD3 was also observed, consistent with TCR engagement following recognition of infected APC (Fig. 3e).

We next evaluated the profile of cytokine released by three $\mathrm{V} \alpha 7.2-\mathrm{J} \alpha 12$ and two $\mathrm{V} \alpha 7.2-\mathrm{J} \alpha 33$ cell clones upon stimulation with E. coli-infected THP-1 cells. Both cell types released Th-0 (granulocyte macrophage colony-stimulating factor), Th-1 (IFN- $\gamma$ and tumour necrosis factor- $\alpha$ (TNF- $\alpha$ )) and Th-2 (interleukin (IL)-4, IL-5, IL-10 and IL-13)-associated cytokines in an antigen-dependent manner, with no major differences in the profile of cytokines produced between the two groups (Fig. 4a). None of the clones released IL-17, while all of them produced soluble CD40L (sCD40L) (Fig. 4a). Importantly, the above cytokines were undetectable in the supernatants of E. coli-infected THP-1 cells cultured in absence of $\mathrm{T}$ cells (Fig. 4b), thus ruling out a possible release by APC. These data suggested that circulating V $\alpha 7.2-\mathrm{J} \alpha 33$ and V $\alpha 7.2-\mathrm{J} \alpha 12$ MAIT cells might contribute to both Th-1 and Th-2 immune responses in peripheral tissues, demonstrating their potential plasticity and immune-regulatory capacity.

The broad array of cytokines released by the T-cell clones suggested that functionally different populations of MAIT cells exist. To test this hypothesis we investigated six intracellular cytokines on MAIT cells ex vivo in multiparametric analysis using mass cytometry ${ }^{33,34}$. In the three tested donors, a large fraction of MAIT cells released MIP- $1 \beta$, IFN- $\gamma$, TNF- $\alpha$ and IL-2 (Fig. $4 c, d$ ), whereas $\mathrm{IL}-4^{+}$and $\mathrm{IL}-10^{+}$cells were less frequent. Cells co-expressing MIP- $1 \beta$ and IFN- $\gamma$, MIP- $1 \beta$ and TNF- $\alpha$, or MIP- $1 \beta$, IFN- $\gamma$ and TNF $\alpha$ were abundant. Cells expressing only MIP- $1 \beta$ were also abundant in two donors, while cells expressing only IFN- $\gamma$ or TNF- $\alpha$ were less represented. All other cytokine combinations were less detected. These findings confirmed that circulating MAIT cells (i) release diverse cytokines, (ii) comprise distinct sub-populations characterized by specific cytokine expression patterns and (iii) display variability of functional sub-populations among individuals.

Tissue distribution of MAIT cells. We next established a semiquantitative qPCR technique to study the relative abundance of $V \alpha 7.2-J \alpha 33$ and $V \alpha 7.2-J \alpha 12$ transcripts in samples from different tissues. Both transcripts were quantified relative to the amount of total TCR C $\alpha$ mRNA in PBMC and biopsies of tissues including the liver, kidney, gut, lung, lymph nodes, tonsil, prostate and ovary. A strong signal for MAIT TCR V $\alpha$ transcripts was detected in PBMC and V $\alpha 7.2-J \alpha 33$ were the predominant MAIT transcripts (Fig. 5a). In the samples analysed, the V $\alpha 7.2-J \alpha 12$ rearrangement was expressed as a variable fraction, ranging between 0.04 and $0.9 \%$ of total TCR C $\alpha$ transcripts (Fig. 5a). Both TCR $\alpha$ MAIT cell transcripts were even more abundant in the liver and in the kidney, with $V \alpha 7.2-\mathrm{J} \alpha 33$ cells being the prevalent subset overall (Fig. 5a). In both organs the percentage of V $\alpha 7.2-\mathrm{J} \alpha 12$ transcripts was larger than in peripheral blood (Fig. 5a,d,e). In one liver and two kidney samples, the V $\alpha 7.2-\mathrm{J} \alpha 12$ were the only transcripts detected (Fig. 5d,e), showing that in some instances, or in some individuals, $\mathrm{V} \alpha 7.2-\mathrm{J} \alpha 12 \mathrm{~T}$ cells may overcome $\mathrm{V} \alpha 7.2$ J $233 \mathrm{~T}$ cells. A similar pattern was observed in biopsies from intestine, in which the V $\alpha 7.2-\mathrm{J} \alpha 12$ transcript was also more commonly expressed (Fig. 5b,g).

Low levels of the two analysed transcripts were detected in lymph nodes and tonsils, where an increase in the relative abundance of V $\alpha 7.2-\mathrm{J} \alpha 12$ transcripts was also noted (Fig. $5 \mathrm{a}, \mathrm{f})$. In ovary biopsies, the two transcripts were both found (Fig. 5b). The $\mathrm{V} \alpha 7.2-\mathrm{J} \alpha 33$ and $\mathrm{V} \alpha 7.2-\mathrm{J} \alpha 12$ rearrangements were detected in three out of four and two out of four analysed samples, respectively, suggesting differences in the relative proportion of MAIT cells in individual samples (Fig. 5b,h). Low levels of Va7.2 transcripts were present in prostate biopsies, with only the $V \alpha 7.2$ J $\alpha 33$ rearrangement detected in two samples out of five individuals (Fig. 5b).

Biopsies from the lung possessed the fewest TCR $\alpha \beta$ cells: in two out of the nine samples in which TCR C $\alpha$ transcripts were evident, we detected a low level of $\mathrm{V} \alpha 7.2-\mathrm{J} \alpha 33$ but not of $\mathrm{V} \alpha 7.2$ $\mathrm{J} \alpha 12$ expression.

Altogether, these findings indicated that the two populations of human MAIT cells are abundant in circulating lymphocytes and in a range of tissues, not only the liver and intestine as previously reported ${ }^{2,22}$, but also in the kidney and, to a minor extent, lymph nodes, tonsils and ovaries. V $\alpha 7.2-\mathrm{J} \alpha 12 \mathrm{~T}$ cells account for a significant fraction of circulating MAIT cells in adult individuals, and in some donors and/or some tissues they may outnumber classical V $\alpha 7.2-J \alpha 33$ MAIT cells.

\section{Discussion}

The specific influence of polymorphic restriction elements and the variety of stimulatory antigens on the composition of the TCR repertoire remains to be defined. The same issue applies to invariant NKT and MAIT cells, which are restricted to nonpolymorphic antigen-presenting molecules and recognize evolutionarily conserved antigens. Oligoclonality is a hallmark of the 
T-cell populations resident in epithelia and the intestine ${ }^{23,35,36}$, and a skewed peripheral $\mathrm{T}$-cell repertoire is present in chronic infections ${ }^{37-39}$, autoimmune diseases ${ }^{40,41}$ and cancer ${ }^{42-44}$.

The recent advances in high throughput RNA deep sequencing (RNAseq) technology have enabled investigation of the TCR repertoire in unprecedented detail in both health and disease ${ }^{45}$. Here we applied the RNAseq technique combined with single
T-cell cloning to systematically investigate the features of the TCR repertoire of human MAIT cells and in parallel to study their function and antigen responsiveness.

We confirmed that the TCR $\beta$ repertoire of MAIT cells is strongly skewed towards $\mathrm{V} \beta 2$ and $\mathrm{V} \beta 13$ chains (TRBV20 and TRBV6 genes), as suggested by studies performed on small numbers of sequences ${ }^{1,21}$. Our study showed a marked a

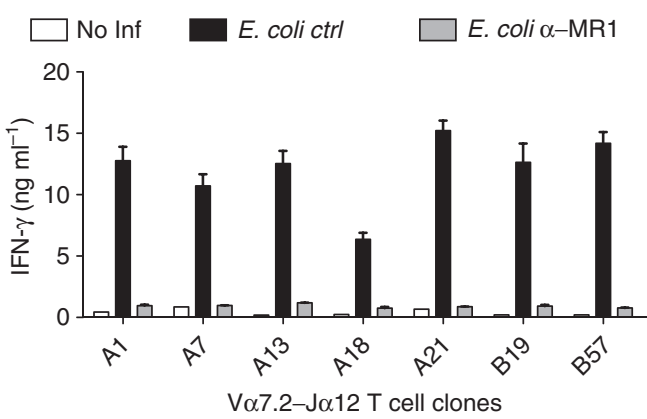

b

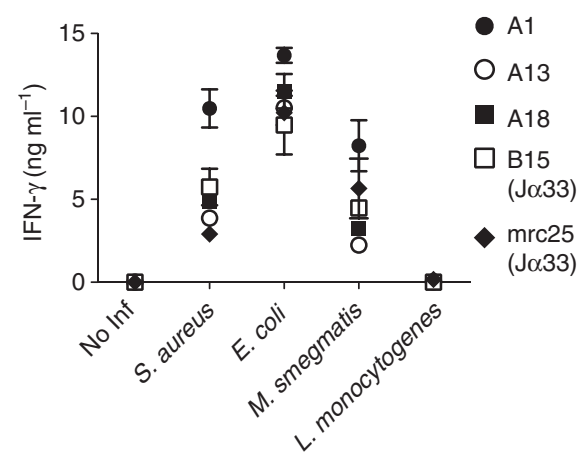

C

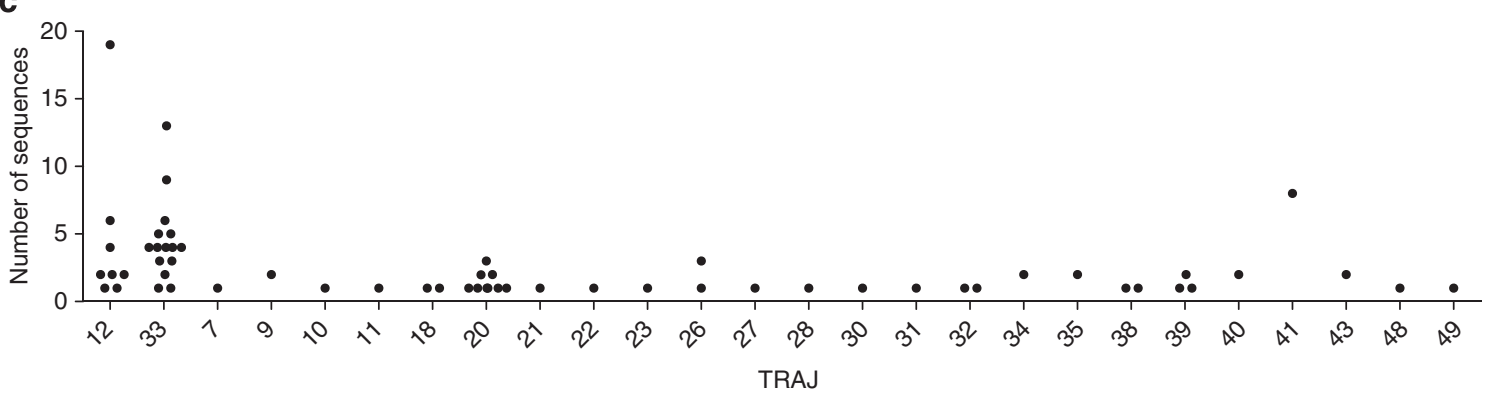

d

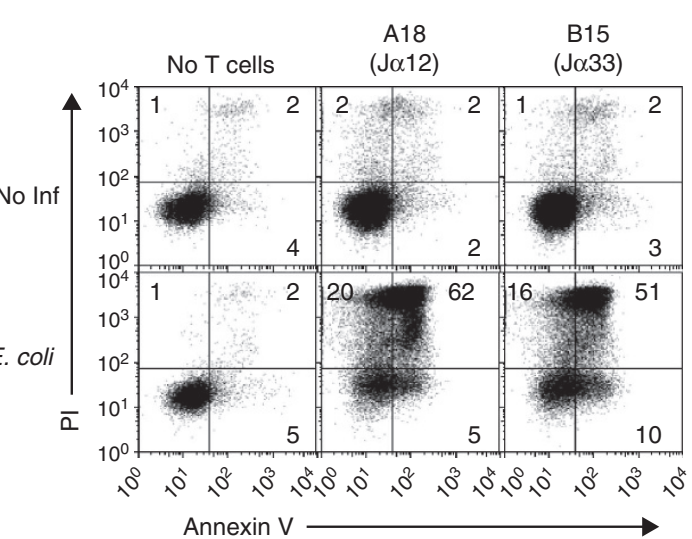

e No APCs --- No Inf - E. coli

f
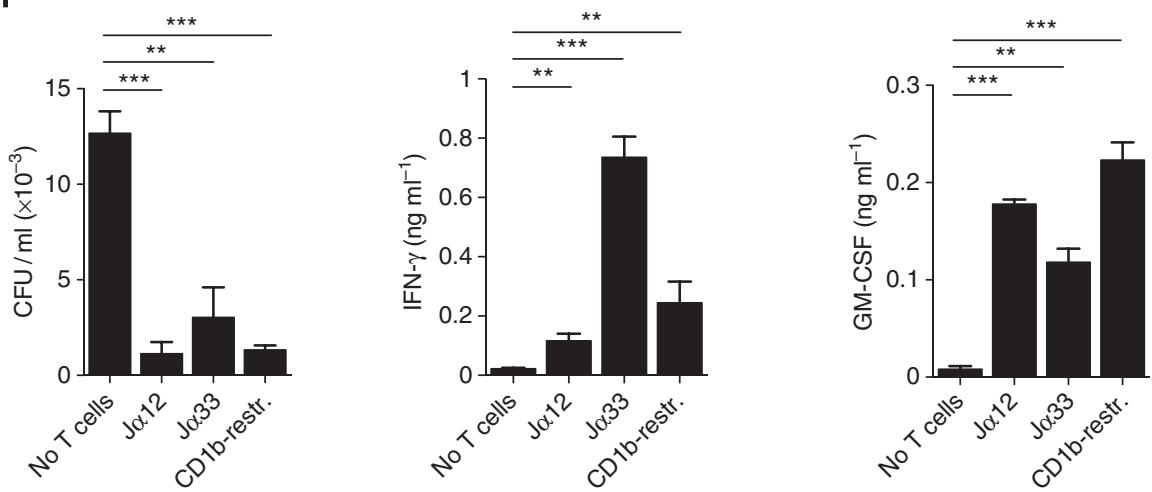
oligoclonality of the CDR3 $\beta$ chains in MAIT cells from peripheral blood and normal liver. Unexpectedly, $5 \%$ of CDR $3 \beta$ amino acid clonotypes covered on average $80 \%$ of the total TCR $\beta$ repertoire in MAIT cells; furthermore, 250 sequences represented $50 \%$ of the repertoire, with frequencies of individual clonotypes reaching up to $12 \%$ of total MAIT- $\beta$ sequences. Such frequencies were much higher than those found in non-MAIT control cell populations, which displayed a more heterogeneous $\mathrm{V} \beta$ usage and a larger number of unique CDR3 $\beta$ clonotypes.

How the extreme oligoclonality of human MAIT cells is generated remains a matter of speculation. The selective expansion of particular clonotypes is probably driven by commensal microbial antigens, as MAIT cells are rare in cord blood, expand early after birth and only then acquire markers of memory $\mathrm{T}$ cells ${ }^{2,13}$. This possibility is supported by the finding that MAIT cells are not detected in germ-free mice but do appear after colonization of the animal by commensal bacteria ${ }^{14}$. Thus, the oligoclonality of the MAIT cell population might be driven by a combination of non-exclusive factors, probably including a high affinity of the expanded TCR heterodimers for MR1-antigen complexes and the presence of an optimal extracellular stimulatory milieu at the site of antigen recognition.

The availability of large numbers of TCR- $\beta$ sequences allowed a detailed analysis of the individual clonotypes. We did not find amino acid motifs within the CDR3 $\beta$, although it does contact the MR1- $\alpha 1$ chain $^{31,32}$. The absence of conserved motifs is consistent with the known absence of effects on antigen reactivity on single amino acid substitutions in the CDR3 $\beta$ chain of MAIT TCR ${ }^{30,46}$. We instead found that MAIT cells displayed a bias in the length of the $\mathrm{CD} 3 \beta$ domain, with the vast majority of the clonotypes

Table 3 | Sequences of V $\alpha 7.2-J \alpha 12$ (TRAV1-2-TRAJ12) and V $\alpha$ 7.2-J $\alpha 20$ (TRAV1-2-TRAJ20) rearrangements obtained by RNA deep-sequencing analysis in different donors.

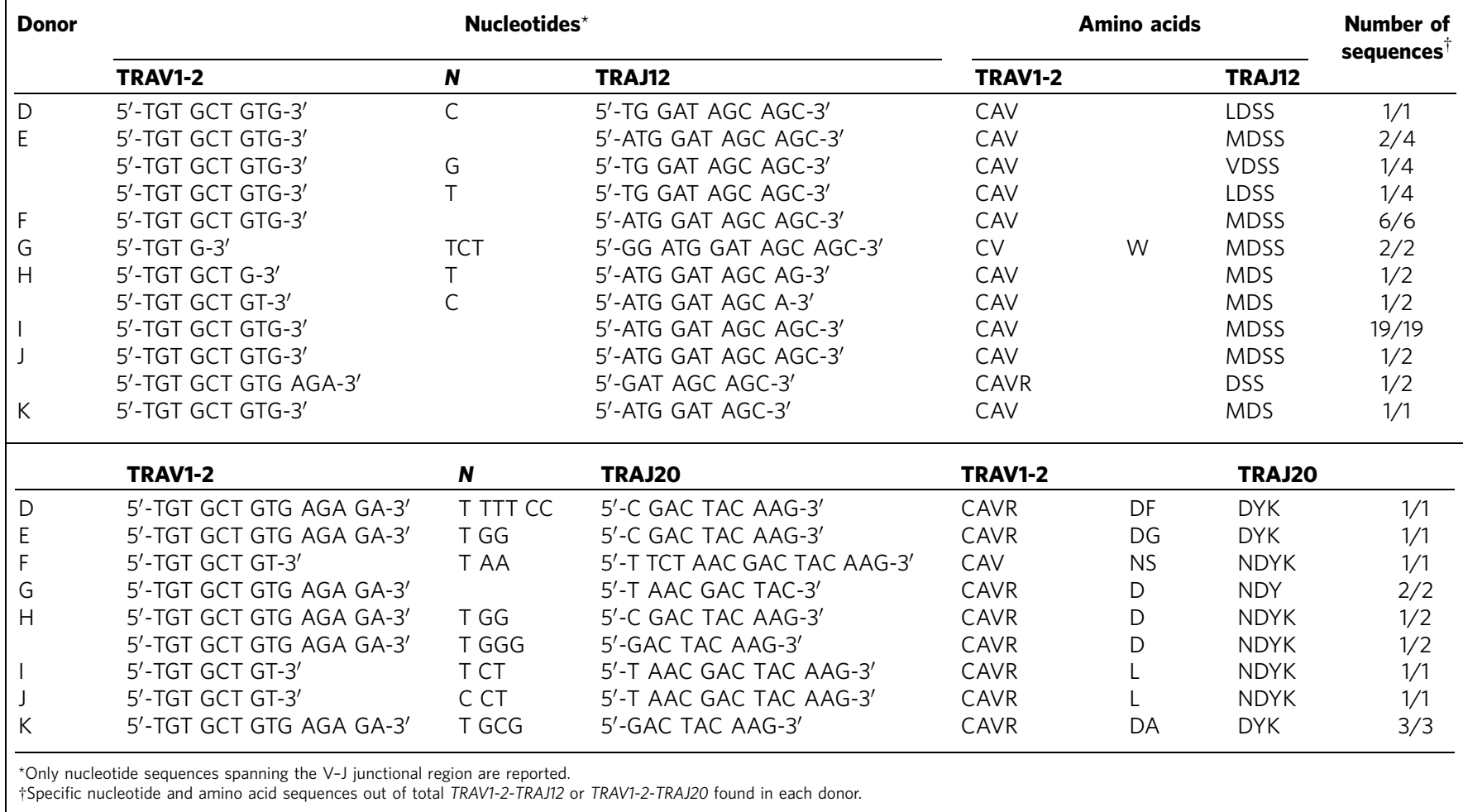

Figure 3 | V $\alpha \mathbf{7 . 2 - J} \alpha 12$ T cells represent a subset of MAIT cells. (a) Seven V $\alpha 7.2-J \alpha 12$ T-cell clones were cultured with THP-1 cells that were either not infected (no Inf), or infected with $E$. coli in the presence of anti-MR1 ( $\alpha$-MR1) or isotype control (ctrl) antibodies. Amount of IFN- $\gamma$ released is shown as mean \pm s.d. of triplicate cultures. Results are representative of four independent experiments. (b) THP-1 cells either not infected (no Inf) or infected with the indicated bacteria were used to stimulate three $\mathrm{V} \alpha$ 7.2-J $\alpha 12$ and two $\mathrm{V} \alpha$ 7.2-J $\alpha 33$ MAIT clones. Points represent the amount of IFN- $\gamma$ released from the indicated T-cell clones and are presented as mean \pm s.d. Results are representative of three independent experiments. (c) Number of sequences in which the $V \alpha 7.2$ gene was found rearranged with individual $J \alpha$ gene segments. Data were generated by deep mRNA sequencing of lymphocyte-enriched populations from 17 donors. Each point represents a single donor. (d) V $\alpha 7.2-J \alpha 12$ and V $\alpha 7$.2-J $\alpha 33$ MAIT cell clones induced apoptosis in E. coli-infected (E. coli) and not infected (no Inf) THP-1 cells. Infected and not infected THP-1 cells were cultured in the absence of T cells (no T cells) as a control. After $24 \mathrm{~h}$, cells were stained with propidium iodide (PI) and Annexin $\mathrm{V}$, and analysed by flow cytometry after gating for CD3 ${ }^{-}$cells. Numbers indicate the percentage of cells in each quadrant. Results are representative of three independent experiments. (e) Expression of surface CD107a and CD3, and intracellular Granzyme B in two MAIT clones upon co-culture with not infected (grey histogram) or E. coli-infected (white histogram) THP-1 cells. T cells were also cultured in the absence of THP-1 cells (dotted histogram). Results are representative of three independent experiments. (f) V $\alpha 7.2-J \alpha 12$ and Va7.2-J 333 MAIT clones, and a CD1b-restricted T-cell clone specific for a mycobacterial lipid antigen (CD1b-restr.) kill intracellular BCG (left panel) and release IFN- $\gamma$ (middle panel) and granulocyte macrophage colony-stimulating factor (GM-CSF; right panel). Results are shown as mean $\pm \mathrm{s}$.d. of triplicate cultures. Colony-forming unit counts and cytokine release were compared with no T-cell group using unpaired Student's $t$-test. ${ }^{\star \star} P \leq 0.001$, ${ }^{\star \star \star} P \leq 0.0001$. 
Table 4 | Phenotype and amino acid TCR $\beta$ sequences of V $\alpha 7.2-J \alpha 12$ T-cell clones from donor $2 B$.

\begin{tabular}{|c|c|c|c|c|c|c|}
\hline \multirow[t]{2}{*}{ T-cell clone } & \multirow[t]{2}{*}{ CD4 } & \multirow[t]{2}{*}{ CD8 } & \multirow[t]{2}{*}{ CD161 } & \multicolumn{3}{|c|}{ TCRß } \\
\hline & & & & V-D-J & CDR3 & CDR3 length \\
\hline 20.7A21 & + & nd & + & BV6-1, BD2, BJ2-5 & ASSDGPAEETQY & 12 \\
\hline $20.7 B 57$ & + & nd & + & BV6-1, BD2, BJ2-5 & ASSDGPAEETQY & 12 \\
\hline $20.7 \mathrm{~A} 1$ & - & nd & + & BV6-4, BD1, BJ2-3 & ASSPGTASTDTQY & 13 \\
\hline $20.7 A 7$ & - & nd & + & BV6-4, BD1, BJ2-3 & ASSPGTASTDTQY & 13 \\
\hline 20.7A13 & - & nd & + & BV6-4, BD1, BJ2-3 & ASSPGTASTDTQY & 13 \\
\hline $20.7 A 18$ & - & nd & + & BV6-4, BD1, BJ2-3 & ASSPGTASTDTQY & 13 \\
\hline
\end{tabular}

a $\square \mathrm{A} 1 \quad \square \mathrm{A} 13 \quad \mathrm{~A} 18 \quad \square \mathrm{B} 15(\mathrm{~J} \alpha 33) \quad \square \mathrm{mrc25}(\mathrm{J} \alpha 33)$
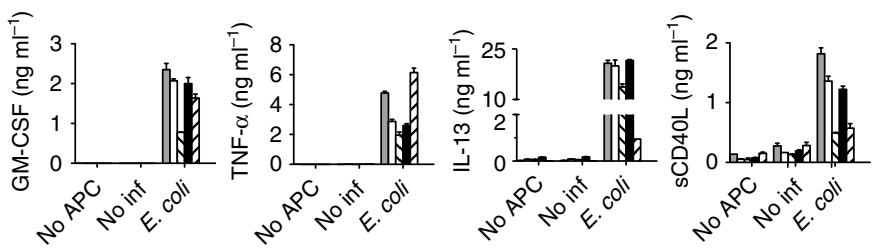

b

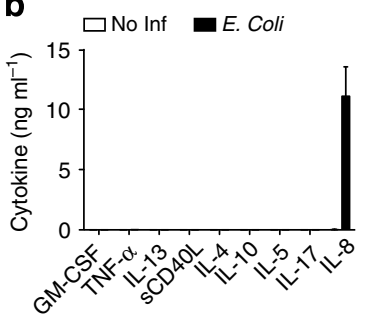

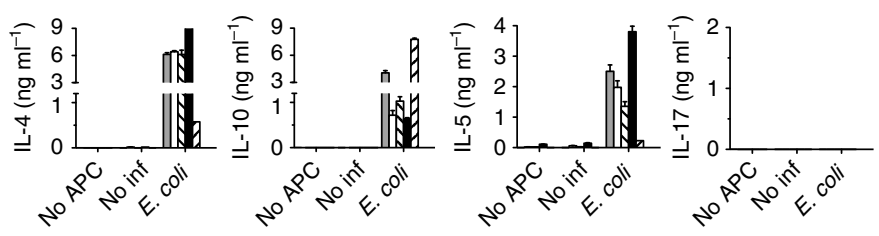

C

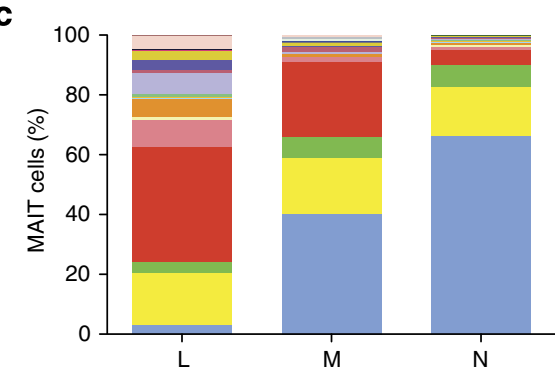

d
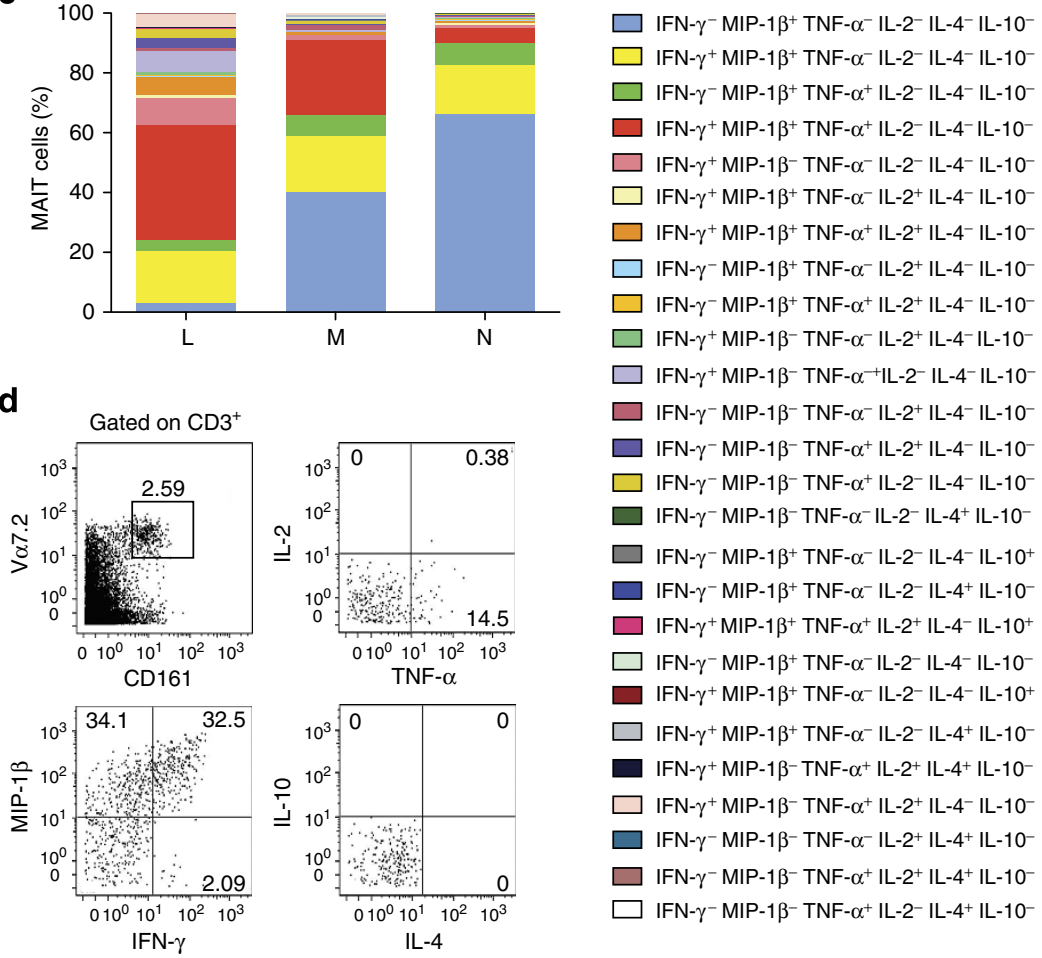

Figure 4 | Cytokine profile of V $\alpha 7.2-J \alpha 12$ and V $\alpha 7.2-J \alpha 33$ MAIT clones, and of ex vivo-stimulated MAIT cells. (a) Multiplex cytokine analysis of $\vee \alpha 7.2-J \alpha 12$ and $V \alpha$ 7.2-J $\alpha 33$ MAIT clones upon co-culture with THP-1 cells either not infected (no Inf) or infected with E. coli (E. coli). Background release of cytokines by unstimulated T cells is also shown (no APC). Data are presented as mean \pm s.d. of duplicate cultures. (b) Cytokine release profile of not infected and E. coli-infected THP-1 cells in the absence of T cells. THP-1 cells did not release any of the measured cytokines except IL-8, which represented a control of the infection. Data are expressed as mean \pm s.d. of duplicate cultures. (c) Summary of mass cytometry analysis of MAIT cell functional capacity. Cumulative frequencies of the most common cytokine combinations produced by stimulated cells are coded by colour for each of the three normal donors $(\mathrm{L}, \mathrm{M}, \mathrm{N}$ ) tested. (d) Dot plots illustrating the mass cytometry analysis in a representative donor. MAIT cells were gated on $\mathrm{CD}^{+}, \mathrm{V} \alpha 7.2^{+}$and $\mathrm{CD} 161^{\text {high }}$, and analysed for intracellular expression of the indicated cytokines. 
a

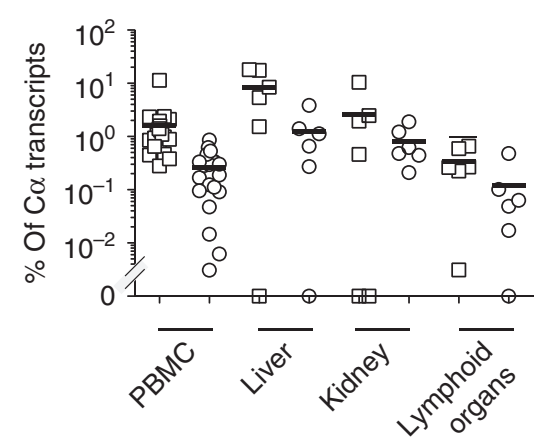

C

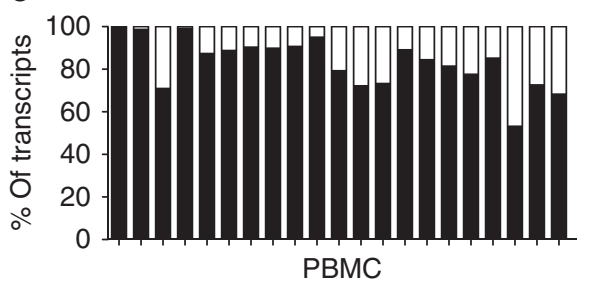

e

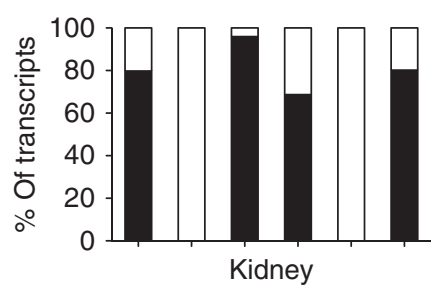

g

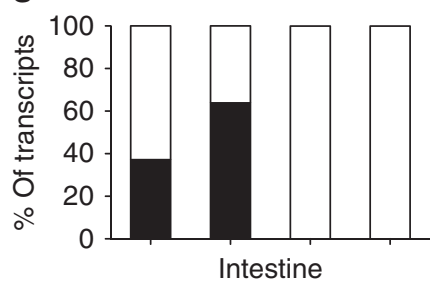

b

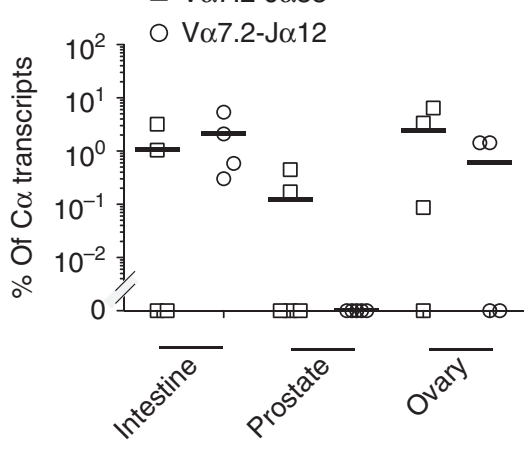

d

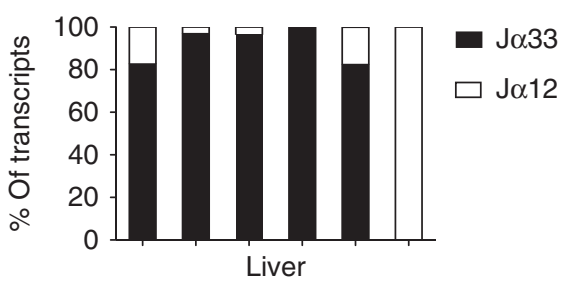

f

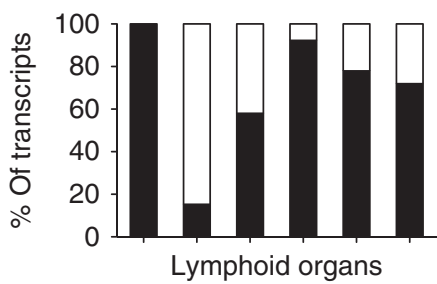

h

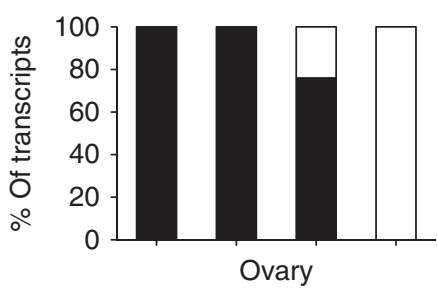

Figure 5 | Tissue distribution of $\mathbf{V} \mathbf{\alpha 7 . 2 - J} \boldsymbol{\alpha} \mathbf{3 3}$ and $\mathbf{V} \mathbf{\alpha 7 . 2 - J} \boldsymbol{\alpha} \mathbf{1 2}$ cells. (a,b) The percentage of $\mathrm{V} \alpha 7.2-J \alpha 12$ and $\vee \alpha 7.2-J \alpha 33$ transcripts among total TCR $\alpha \beta$ transcripts in the indicated tissues was assessed by quantitative PCR (qPCR) by quantifying the amount of $V \alpha 7.2-J \alpha 12$ and $V \alpha 7.2-J \alpha 33$ amplicons relative to that of TCR C $\alpha$ amplicon. Horizontal bars indicate the mean value. Relative proportions of V $\alpha 7.2-J \alpha 33$ and V $\alpha 7.2-J \alpha 12$ transcripts in (c) PBMC, (d) liver, (e) kidney, (f) lymphoid organs (tonsils, columns 1 and 2, and lymph nodes, columns 3-6), ( $($ ) intestine (colon) and (h) ovary. Relative proportions of $V \alpha 7.2-J \alpha 12$ and $V \alpha 7.2-J \alpha 33$ transcripts were derived from the qPCR analysis, that is, $\%$ of $V \alpha 7.2-J \alpha 12$ transcripts $=\%$ of $V \alpha 7.2-J \alpha 12 /(\%$ of $\vee \alpha 7.2-J \alpha 12+V \alpha 7.2-J \alpha 33)$.

ranging between 11 and 14 amino acids long. This length might be preferentially selected for optimal chain pairing and/or docking on the $\alpha 1$ helix of MR1 (refs 31,32).

The MAIT lymphocyte repertoire was also found to be stable over time, at least across the 5-month interval studied. Thus, the TCR repertoire of MAIT cells is both markedly oligoclonal and stable.

A second key finding of our study is the definition of a novel population of MAIT cells expressing an invariant TCR chain and characterized by the $\mathrm{V} \alpha 7.2-\mathrm{J} \alpha 12$ rearrangement. These cells shared fundamental traits with 'conventional' V $\alpha 7.2-J \alpha 33$ MAIT cells: (i) they were contained in the MAIT population defined as $\mathrm{V} \alpha 7.2^{+} \mathrm{CD} 161^{\text {high }}$, (ii) they were oligoclonal and (iii) they reacted in an MR1-dependent manner only to riboflavinproducing bacteria ${ }^{15}$.
Deep RNA sequencing also detected a small number of V $\alpha 7.2-J \alpha 20$ sequences, previously found to be expressed by rare MAIT cells ${ }^{10}$. These rearrangements were less abundant than $\mathrm{V} \alpha 7.2-\mathrm{J} \alpha 33$ and $\mathrm{V} \alpha 7.2-\mathrm{J} \alpha 12$, indicative of a third population of MAIT cells. Our data are in agreement with a recent study in which the $\mathrm{V} \alpha 7.2-\mathrm{J} \alpha 12$ and $\mathrm{V} \alpha 7.2-\mathrm{J} \alpha 20$ chains were found expressed in MAIT cells identified by MR1-tetramer staining ${ }^{21}$.

The analysis of the relative abundance of $\mathrm{V} \alpha 7.2-\mathrm{J} \alpha 33$ and $\mathrm{V} \alpha 7.2-\mathrm{J} \alpha 12$ transcripts within the tissue samples revealed a predominance of the $\mathrm{V} \alpha 7.2-\mathrm{J} \alpha 12 \mathrm{~T}$ cells in solid tissues of some individuals, raising the possibility of differences in tissue-homing capacity between the two MAIT cell populations. In some tissues, it appeared that $\mathrm{V} \alpha 7.2-\mathrm{J} \alpha 12 \mathrm{~T}$ cells were more abundant than $\mathrm{V} \alpha 7.2-\mathrm{J} \alpha 33 \mathrm{~T}$ cells. Whether this differential tissue distribution reflects responsiveness to different types of antigens with unique 
tissue localization and/or to a different infection history of each donor warrants further investigations. The fact that both MAIT cell populations were present within the kidney and, albeit to a lesser extent, in ovaries, prostate and peripheral lymphoid tissues is interesting in its own right, as these organs are normally sterile in healthy individuals. The question now arises whether MAIT cells accumulate in these tissues following local recognition of microbial antigens, or instead are perhaps autoreactive and recognize antigens specific to these sites.

The pattern of cytokines released by $\mathrm{V} \alpha 7.2-\mathrm{J} \alpha 33$ and $\mathrm{V} \alpha 7.2$ $\mathrm{J} \alpha 12$ MAIT cell clones in response to $E$. coli was comparable and was characterized by large amounts of Th0, Th1 and Th2 cytokines, and sCD40L. Both types of MAIT cell efficiently killed E. coli-infected APC and also promoted killing of intracellular mycobacteria. The cytotoxic activity of MAIT cells against infected cells was in accordance with a primary role in controlling pathogen invasion: indeed, in mice infected with Francisella tularensis, MAIT cells accumulate in the lungs and release IFN- $\gamma$, $\mathrm{TNF} \alpha$ and IL-17A ${ }^{47}$

In addition, their capacity to release regulatory cytokines after bacterial recognition also suggested that MAIT cells might be able to modulate the antimicrobial function of other immune cells, as described for iNKT cells ${ }^{11}$.

Perhaps surprisingly, our MAIT cell clones did not release IL17 in response to bacterially infected APC, reflecting the fact that antigenic stimulation via the TCR is not sufficient to induce a Th17 -like function in these cells ${ }^{2,22}$. Instead, activation of this capacity probably requires additional environmental signals, one of which is likely to be IL-7 (ref. 22). Multiparametric mass cytometry identified MAIT cell sub-populations co-expressing different cytokines. Most of them produced ex vivo the chemokine MIP-1 $\beta$ (CCL4), which is mostly released by perforin-low memory $\mathrm{T}$ cells and facilitates attraction of NK cells, monocytes and other immune cells. Thus, MAIT cells might participate in recruiting other cells to the site of infection and promoting local inflammation.

In conclusion, we have shown that MR1-restricted $\mathrm{T}$ cells, which use at least two different TCR V $\alpha 7.2-\mathrm{J} \alpha$ chains, exhibit a stable and unexpectedly oligoclonal repertoire in the blood of healthy humans. Their abundance in solid tissues, reactivity to microbial compounds and release of MIP-1 $\beta$ is suggestive of their local protective and immunoregulatory functions.

\section{Methods}

Cells, bacteria and mAbs. The following cell lines were used as APC: THP-1, Daudi (both from American Type Culture Collection), Daudi MR1- $\beta 2 \mathrm{~m}$ (described below), monocyte-derived dendritic cell (obtained as described ${ }^{48}$ ). APC were infected with either E. coli (DH5 $\alpha$, Invitrogen), S. aureus (MW2, MnCop, LAC, MRSA252, COL), M. smegmatis ( $\mathrm{mc}^{2} 155$, NCTC 8 159) or L. monocytogenes (EGD).

The following mAbs specific to human determinants were used for flow cytometry $\left(5 \mu \mathrm{g} \mathrm{ml}^{-1}\right)$ : CD4-Pacific blue (PB) (BioLegend) or -fluorescein isothiocyanate (Caltag), CD161-PERCP/Cy5 or -Alexa Fluor 647 (BioLegend, clone HP-3G10), Va7.2-PE (BioLegend, clone 3C10), CD107a (LAMP-1)-biotin (BioLegend, clone H4A3), CD69-PB (BioLegend, clone FN50), CD3-PB or -PE/ Cy7 (BioLegend, clone UCHT1), Granzyme B-fluorescein isothiocyanate (Bio Legend, clone GB11), CD8-PB (Dako) or -PE (BioLegend), AnnexinV-APC (Becton Dickinson), TCRV $\alpha 24$-biotin (C15) TCRV 2 -biotin (4G6), MHC Iunlabelled (W6/32, mIgG2a). Biotinylated mAbs were revealed with streptavidin$\mathrm{PE},-$ Alexa Fluor 488 or -Brilliant violet $421\left(2 \mu \mathrm{g} \mathrm{ml}{ }^{-1}\right.$, BioLegend). MR1-specific mAbs 26.5 and 12.2 (both mouse IgG2a) were provided by Ted Hansen, James McCluskey, Marina Cella and Marco Colonna ${ }^{17}$. For flow cytometric experiments, unlabelled mAbs (W6/32, 26.5 and 12.2) were revealed with goat-anti mouse $\operatorname{IgG} 2 \mathrm{a}-\mathrm{PE}\left(2 \mu \mathrm{g} \mathrm{ml}^{-1}\right.$, Southern Biotech).

Human samples. Human intestine (colon), lung, kidney, ovary, testis, liver, tonsil and lymph node (submandibular and axillary) biopsy samples were obtained from the Pathology Department, University Hospital Basel. The study was approved by the Ethics Committee of Basel (EK: 142/13). Heparinized blood was obtained from healthy donors (age 20-35 years) and PBMC isolated using Ficoll-Paque gradients
(Axis-Shield PoC AS). The study was approved by the Ethics Committee of the National University of Singapore (NUS-IRB 09-256 and NUS-IRB 10-250).

Liver-associated mononuclear cells were collected from living healthy donors after portal flush using cold preservation solution following removal of the right lobe of donor's liver. Collection was performed according to the standard protocol preceding liver transplantation ${ }^{22}$. The study was approved by the Ethics Committee of Gleaneagle Hospital, Parkway Health Group, Singapore (PIEC/2012/037).

All donors gave written informed consent.

Flow cytometry. Cell surface labelling was performed according to standard procedures. Intracellular labelling was performed following cell fixation in $2 \%$ paraformaldehyde and permeabilization with $0.5 \%$ saponin.

To measure killing of infected or non-infected APC by T cells, samples were stained with propidium iodide and labelled with anti-CD3 and anti-Annexin mAbs after $24 \mathrm{~h}$ of co-culture. For the analysis of CD107a, Granzyme B and CD3 expression, samples were labelled with mAbs specific for these antigens after $4 \mathrm{~h}$ of co-culture.

Samples were analysed by CyAn ADP flow cytometer (DakoCytomation), with gating to exclude doublets and non-viable cells on the basis of pulse width and incorporation of propidium iodide. Data were analysed using FloJo (TreeStar).

Sorting of PBMC and liver T cells from healthy donor samples were carried out using the BD Influx Cell Sorter with BD FACS Software v1.0.0.650 (BD Biosciences).

MAIT cell cloning. T-cell clones were established from PBMC of normal donors as reported $^{49}$. CD ${ }^{+} \mathrm{CD} 4^{-} \mathrm{V} \alpha 24^{-} \mathrm{V} \delta 2^{-} \mathrm{CD} 161^{\text {high }}$ cells were sorted from Donor 1 (Supplementary Fig. 1a). For other donors, V $\alpha 7 \cdot 2^{+} \mathrm{CD} 161^{\text {high }}$ cells were sorted using anti-V $\alpha 7.2$ and anti-CD161 mAbs as illustrated in Supplementary Fig. 2. Sorted cells were cloned by limiting dilution using phytohemagglutinin $\left(1 \mu \mathrm{g} \mathrm{ml}^{-1}\right)$ (Wellcome Laboratories), human rIL-2 (100 $\mathrm{U} \mathrm{ml}^{-1}$, Hoffmann-La Roche) and irradiated PBMC $\left(5 \times 10^{5} \mathrm{ml}^{-1}\right)$. T-cell clones were re-stimulated periodically following the same protocol.

Daudi cells expressing MR1A covalently linked with $\mathbf{\beta 2} \mathbf{m}$. A human MR1A complementary DNA construct linked to $\beta 2 \mathrm{~m}$ via a flexible Gly-Ser linker was generated by PCR using the following primers: Link-MR1-F, 5'-GCGGAGGTGGC AGTGAACGGACGCACTCTCTGAGATATTTTCG-3'; NotI-MR1-cyt-R, 5'-GCG GCCGCTCATCGATCTGGTGTTGGAAGG and XhoI- $\beta 2 \mathrm{~m}-\mathrm{F}, 5^{\prime}$-CTCGAGAT GTCTCGCTCCGTGGCCTTA- ${ }^{\prime}$; Link- $\beta 2 \mathrm{~m}-\mathrm{R}, 5^{\prime}$-AACCTCCTCCACCCATGTC TCGATCCCACTTA- $3^{\prime}$ and Link F, $5^{\prime}$-GAGACATGGGTGGAGGAGGTTCTG GAGGCGGTGGCAGTG- $3^{\prime}$; Link R, 5'-GTTCACTGCCACCTCCGCCACTGCC ACCGCCTCCAG- $3^{\prime}$. The $\beta 2 \mathrm{~m}$-linker and linker-MR1A were joined together using two-step splicing with overlap extension PCR and the resulting construct subcloned into the XhoI/NotI sites of the BCMGSNeo expression vector. Daudi cells were transfected by electroporation and selected using G418 (Calbiochem).

Killing of intracellular bacteria. THP-1 cells were infected with Mycobacterium bovis BCG Pasteur strain (multiplicity of infection $=1: 5$ ) for $4 \mathrm{~h}$, washed extensively to remove extracellular bacteria and T cells (APC:T cell ratio $=1: 1$ ) were added. Antibiotic-free medium was used. After $24 \mathrm{~h}$, culture supernatants were harvested for cytokine analysis, and cells were pelleted and lysed with $0.06 \%$ SDS for $10 \mathrm{~min}$ at room temperature. BCG colony-forming units were counted after 3 weeks by plating serial dilutions on Middlebrook 7H11 agar plates supplemented with $10 \%$ oleic-acid-albumin-dextrose-catalase (Difco), in triplicates.

Antigen presentation assays. APC were incubated with bacteria at a multiplicity of infection of 10 for $3 \mathrm{~h}$ at $37^{\circ} \mathrm{C}$ in medium without antibiotics, then washed, incubated in medium with kanamycin $\left(100 \mu \mathrm{g} \mathrm{ml}^{-1}\right)$, gentamycin $\left(10 \mu \mathrm{g} \mathrm{ml}^{-1}\right)$ and ciprofloxacin $\left(10 \mu \mathrm{g} \mathrm{ml}^{-1}\right)$ for $1 \mathrm{~h}$, washed again and plated $\left(5 \times 10^{4}\right.$ per well). For inhibition assays, anti-MR1 mAbs $(26.5)$ were added $\left(20 \mu \mathrm{g} \mathrm{ml}^{-1}\right)$ to APC after infection and cells were incubated for $1 \mathrm{~h}$ before addition of $\mathrm{T}$ cells $\left(10^{5}\right.$ per well). Where indicated, APC not infected or infected with $E$. coli were also cultured in absence of T cells as control. Cytokines released in the supernatants after $24 \mathrm{~h}$ were measured by ELISA or by Milliplex Map Kit Human cytokine/chemokine panel I (Millipore)

Mass cytometry analysis of cytokine production. Cryopreserved human PBMC obtained from three normal donors were thawed and rested overnight before stimulation with phorbol myristate acetate $\left(150 \mathrm{ng} \mathrm{ml}^{-1}\right.$, Sigma Aldrich) and ionomycin $\left(1 \mu \mathrm{M}\right.$, Sigma Aldrich) for $3 \mathrm{~h}$ at $37^{\circ} \mathrm{C}$ in the presence of brefeldin A (eBioscience) and monensin (BioLegend) to probe for intracellular cytokine production using mass cytometry, performed as previously described using DVS/Fluidigm C5 CyTOF ${ }^{34}$. Surface staining was performed on live cells before fixation, permeabilization with permeabilization buffer (BioLegend) and intracellular cytokine staining. Heavy metal conjugated mAbs specific for the following molecules were used to identify MAIT cells and delineate their functional capacities: CD3 (clone UCHT1, $3 \mu \mathrm{g} \mathrm{ml}^{-1}$, Becton Dickinson), V $\alpha 7.2$ (clone 3C10, 
$10 \mu \mathrm{g} \mathrm{ml}^{-1}$, BioLegend), CD161 (clone HP-3G10, $10 \mu \mathrm{g} \mathrm{ml}^{-1}$, BioLegend), IFN- $\gamma$ (clone 4S.B3, $6 \mu \mathrm{g} \mathrm{ml}^{-1}$, BioLegend), MIP-1 $\beta$ (clone D21-1351, $7 \mu \mathrm{g} \mathrm{ml}^{-1}$, BD Pharmingen), TNF- $\alpha$ (clone MAb11, $4 \mu \mathrm{g} \mathrm{ml}^{-1}$, BioLegend), IL-2 (clone MQ1-17H12, $4 \mu \mathrm{g} \mathrm{ml}^{-1}$, BioLegend), IL-4 (clone MP4-25D2, $5 \mu \mathrm{g} \mathrm{ml}^{-1}$, BioLegend), IL-10 (clone JES3-12G8, $10 \mu \mathrm{g} \mathrm{ml}^{-1}$, eBiosciences).

MAIT cell clone TCR sequencing. RNA from MAIT cell clones was prepared using the NucleoSpin RNA II Kit (Macherey Nagel) and cDNA synthesized with Superscript III reverse transcriptase (Invitrogen). MAIT cell clone TCRV $\beta$ were amplified using a commercial TCRV $\beta$ typing amplimer kit (Clontech) as described ${ }^{50}$. MAIT cell clone TCR $\alpha$ were amplified using V $\alpha 7.2$ (5'-GTCGGTCTA AAGGGTACAGT- $\left.3^{\prime}\right)$ and $C \alpha\left(5^{\prime}\right.$-TTTAGAGTCTCTCAGCTGGTA- $\left.3^{\prime}\right)$ primers. PCR-amplified fragments were purified from agarose gel and sequenced.

Next-generation sequencing. For TCR $\alpha$ and $-\beta$ high-throughput sequencing, RNA was prepared after TRIzol lysis of sorted cell populations without in vitro culture. Four populations sorted from six donors according to V $\alpha 7.2$ and CD161 expression were used for TCR $\beta$ studies. Total lymphocytes sorted from PBMC of 17 donors were used for TCR $\alpha$ studies.

TCR $\beta$ analysis: CDR3 $\beta$ regions were amplified and sequenced by Adaptive Biotechnologies Corp. (Seattle, WA) using the ImmunoSEQ assay (http:// www.immunoseq.com). Briefly, a multiplex PCR system was used to amplify CDR3 $\beta$ sequences from cDNA samples using 52 forward primers for the $V \beta$ gene segment and 13 reverse primers for the $J \beta$ segment. This approach generates a 60-bp fragment capable of identifying the VDJ region spanning each unique $\mathrm{CDR} 3 \beta^{51}$. Amplicons were sequenced using the Illumina HiSeq platform. Using a baseline developed from a suite of synthetic templates ${ }^{52}$, primer concentrations and computational corrections were used to correct for the primer bias common to multiplex PCR reactions. Raw sequence data were filtered based on the TCR $\beta$ V, D and J gene definitions provided by the International Immunogenetics Information (IMGT, www.imgt.org) database and binned using a modified nearest-neighbour algorithm to merging closely related sequences and remove both PCR and sequencing errors. Data were analysed using the ImmunoSEQ analyser toolset. This approach is capable of detecting one cell in 40,000 T cells using the Survey level, or one cell in 200,000 using the Deep level ${ }^{53}$

TCR $\alpha$ analysis: cDNA libraries were prepared from total RNA. ERCC RNA Spike-In Controls (Life Technologies) were added to the reaction according to manufacturer's recommendations. The fragmented mRNA samples were subjected to cDNA synthesis using Illumina TruSeqTM RNA sample preparation kit using multiplexing indexes. The pooled cDNA libraries were subjected to a indexed PE sequencing run of $2 \times 51$ cycles on an Illumina HiSeq 2000. Using this strategy, 30 to 40 millions of paired-end reads were produced for each sample.

TCR $V \alpha$ and $J \alpha$ region annotation was taken from IMGT and converted to HG19 co-ordinates with UCSC liftover (http://genome.ucsc.edu/cgi-bin/ hgLiftOver). RNAseq fastq paired-end data were first quality checked with fastqc (http://www.bioinformatics.babraham.ac.uk/projects/fastqc/) and then mapped to an ERCC spike in database with Bowtie, using default parameters except maxinsert-size set to 500. Picard tools (http://picard.sourceforge.net/) were used to determine the mean mate distance and s.d. for each sample. The data were then mapped to HG19 with TopHat2 (http://tophat.cbcb.umd.edu, using default parameters except for the mate information, UCSC annotation as a guide (http:// genome.ucsc.edu/cgi-bin/hgTables?hgsid=337339223) and allowing novel junctions. The resulting BAM files and the SAM flags within them were then interrogated with SAMtools (http://samtools.sourceforge.net/, to find all reads that mapped in a forward direction on any of the TCR V regions, but for which the mate pair was unmapped (under the assumption this mate pair was mapping across the V-J junction). The unmapped sequences from the TopHat2 mapping were then searched by name to find the sequence for these associated unmapped reads.

A synthetic reference database was constructed, which consisted of all possible $\mathrm{V}$ and $\mathrm{J}$ region fusions, and indexed with Bowtie2. Bowtie2 was then run on this database using the associated unmapped reads with relaxed gap opening/extension penalties (command line parameters - rdg 4,1-rfg 4,1). A custom script was written, which integrated the resulting BAM file and the CIGAR string within it, with the HG19-converted V and J region annotation from IMGT, to produce an alignment file that displayed these reads in the context of the V-J junction. All of this methodology was written within the Pipeline Pilot frame work (www.accelrys.com).

Real-time PCR for MAIT cell populations. Real-time PCR was performed in a 20$\mu \mathrm{l}$ reaction volume containing $0.5 \mu \mathrm{M}$ of each primer and $1 \mu \mathrm{l}$ of cDNA in Power SYBRgreen MasterMix (Applied Biosystems). For each sample, three reactions were run for $\mathrm{V} \alpha 7.2-\mathrm{J} \alpha 33$ and $\mathrm{V} \alpha 7.2-\mathrm{J} \alpha 12$ TCR rearrangements and the constant region of TCR $\alpha$. The following running method was used for PCR: initial incubation for $2 \mathrm{~min}$ at $50^{\circ} \mathrm{C}$, incubation for $10 \mathrm{~min}$ at $95^{\circ} \mathrm{C}, 40$ cycles of $15 \mathrm{~s}$ at $95^{\circ} \mathrm{C}$ and $1 \mathrm{~min}$ at $60^{\circ} \mathrm{C}$. For the MAIT-cell V-J region-specific and total C-alpha reactions, the following primers were used: V $\alpha 7.2 J \alpha 33$ _1Fwd, $5^{\prime}$-GTCGGTCTA AAGGGTACA-3', V $\alpha 7.2 J \alpha 33$ _Rev, 5'-CCAGATTAACTGATAGTTGCTA; V $\alpha 7.2 J \alpha 12 \_1 F w d, 5^{\prime}$-AGTCGGTCTAAAGGGTACAGTT-3', V $\alpha 7.2 J \alpha 12 \_1$ Rev,
5'-GGTCCCACTCCCGAAGAT; C $\alpha \_1$ Fwd, 5 -ACGCCTTCAACAACAGCA TTA- $3^{\prime}, C \alpha \_1$ Rev, $5^{\prime}$-TCAGGAGGAGGATTCGGAAC- $3^{\prime}$. Reactions were run on an ABI 7500 real-time PCR System (Applied Biosystems). Numbers of transcripts were calculated according to the standard curve generated using a cloned fulllength $T C R \alpha$ gene (V $\alpha 7.2-\mathrm{J} \alpha 33$ and $V \alpha 7.2-\mathrm{J} \alpha 12)$. Results for both populations are represented as a percent of total $\mathrm{T}$ cells.

Statistical analyses. For TCR $\beta$ sequence analysis, data were analysed with MannWhitney $U$-test and unpaired Student's $t$-test with Welch's correction. For cytokine and colony-forming unit assays, data were analysed with a two-tailed unpaired Student's $t$-test with Welch's correction. For staining assays, data were analysed with Mann-Whitney-Wilcoxon multiple-comparison test.

\section{References}

1. Tilloy, F. et al. An invariant $\mathrm{T}$ cell receptor alpha chain defines a novel TAP-independent major histocompatibility complex class Ib-restricted alpha/beta T cell subpopulation in mammals. J. Exp. Med. 189, 1907-1921 (1999).

2. Dusseaux, M. et al. Human MAIT cells are xenobiotic-resistant, tissue-targeted, CD161hi IL-17-secreting T cells. Blood 117, 1250-1259 (2011).

3. Kawachi, I., Maldonado, J., Strader, C. \& Gilfillan, S. MR1-restricted V alpha 19i mucosal-associated invariant $\mathrm{T}$ cells are innate $\mathrm{T}$ cells in the gut lamina propria that provide a rapid and diverse cytokine response. J. Immunol. 176, 1618-1627 (2006).

4. Treiner, E. et al. Selection of evolutionarily conserved mucosal-associated invariant T cells by MR1. Nature 422, 164-169 (2003).

5. Gold, M. C. et al. Human mucosal associated invariant $\mathrm{T}$ cells detect bacterially infected cells. PLoS Biol. 8, e1000407 (2010).

6. Le Bourhis, L. et al. Antimicrobial activity of mucosal-associated invariant T cells. Nat. Immunol. 11, 701-708 (2010).

7. Chua, W. J. \& Hansen, T. H. Bacteria, mucosal-associated invariant T cells and MR1. Immunol. Cell Biol. 88, 767-769 (2010).

8. Le Bourhis, L. et al. Mucosal-associated invariant T cells: unconventional development and function. Trends Immunol. 32, 212-218 (2011).

9. Georgel, P., Radosavljevic, M., Macquin, C. \& Bahram, S. The nonconventional MHC class I MR1 molecule controls infection by Klebsiella pneumoniae in mice. Mol. Immunol. 48, 769-775 (2011).

10. Gold, M. C. \& Lewinsohn, D. M. Co-dependents: MR1-restricted MAIT cells and their antimicrobial function. Nat. Rev. Microbiol. 11, 14-19 (2013).

11. Bendelac, A., Savage, P. B. \& Teyton, L. The biology of NKT cells. Annu. Rev Immunol. 25, 297-336 (2007).

12. Martin, E. et al. Stepwise development of MAIT cells in mouse and human. PLoS Biol. 7, e54 (2009).

13. Gold, M. C. et al. Human thymic MR1-restricted MAIT cells are innate pathogen-reactive effectors that adapt following thymic egress. Mucosal Immunol. 6, 35-44 (2012).

14. Treiner, E. et al. Mucosal-associated invariant T (MAIT) cells: an evolutionarily conserved T cell subset. Microbes Infect. 7, 552-559 (2005).

15. Kjer-Nielsen, L. et al. MR1 presents microbial vitamin B metabolites to MAIT cells. Nature 491, 717-723 (2012).

16. Huang, S. et al. Evidence for MR1 antigen presentation to mucosal-associated invariant T cells. J. Biol. Chem. 280, 21183-21193 (2005).

17. Miley, M. J. et al. Biochemical features of the MHC-related protein 1 consistent with an immunological function. J. Immunol. 170, 6090-6098 (2003).

18. Huang, S. et al. MR1 uses an endocytic pathway to activate mucosal-associated invariant T cells. J. Exp. Med. 205, 1201-1211 (2008).

19. Goldfinch, N. et al. Conservation of mucosal associated invariant T (MAIT) cells and the MR1 restriction element in ruminants, and abundance of MAIT cells in spleen. Vet. Res. 41, 62 (2010).

20. Greenaway, H. Y. et al. NKT and MAIT invariant TCRalpha sequences can be produced efficiently by VJ gene recombination. Immunobiology 218, 213-224 (2013).

21. Reantragoon, R. et al. Antigen-loaded MR1 tetramers define T cell receptor heterogeneity in mucosal-associated invariant T cells. J. Exp. Med. 210, 2305-2320 (2013).

22. Tang, X. Z. et al. IL-7 licenses activation of human liver intrasinusoidal mucosal-associated invariant T cells. J. Immunol. 190, 3142-3152 (2013).

23. Van Kerckhove, C. et al. Oligoclonality of human intestinal intraepithelial T cells. J. Exp. Med. 175, 57-63 (1992).

24. Regnault, A., Kourilsky, P. \& Cumano, A. The TCR-beta chain repertoire of gut-derived T lymphocytes. Semin. Immunol. 7, 307-319 (1995).

25. Exley, M., Garcia, J., Balk, S. P. \& Porcelli, S. Requirements for CD1d recognition by human invariant Valpha24+ CD4-CD8- T cells. J. Exp. Med. 186, 109-120 (1997).

26. Burk, M. R., Mori, L. \& De Libero, G. Human V gamma 9-V delta 2 cells are stimulated in a cross-reactive fashion by a variety of phosphorylated metabolites. Eur. J. Immunol. 25, 2052-2058 (1995). 
27. Arden, B., Clark, S. P., Kabelitz, D. \& Mak, T. W. Human T-cell receptor variable gene segment families. Immunogenetics 42, 455-500 (1995).

28. Folch, G. \& Lefranc, M. P. The human T cell receptor beta diversity (TRBD) and beta joining (TRBJ) genes. Exp. Clin. Immunogenet. 17, 107-114 (2000).

29. Turner, S. J., Doherty, P. C., McCluskey, J. \& Rossjohn, J. Structural determinants of T-cell receptor bias in immunity. Nat. Rev. Immunol. 6, 883-894 (2006).

30. Reantragoon, R. et al. Structural insight into MR1-mediated recognition of the mucosal associated invariant T cell receptor. J. Exp. Med. 209, 761-774 (2012).

31. Lopez-Sagaseta, J. et al. The molecular basis for Mucosal-Associated Invariant T cell recognition of MR1 proteins. Proc. Natl Acad. Sci. USA 110, E1771-E1778 (2013).

32. Patel, O. et al. Recognition of vitamin B metabolites by mucosal-associated invariant T cells. Nat. Commun. 4, 2142 (2013).

33. Bandura, D. R. et al. Mass cytometry: technique for real time single cell multitarget immunoassay based on inductively coupled plasma time-of-flight mass spectrometry. Anal. Chem. 81, 6813-6822 (2009).

34. Newell, E. W., Sigal, N., Bendall, S. C., Nolan, G. P. \& Davis, M. M. Cytometry by time-of-flight shows combinatorial cytokine expression and virus-specific cell niches within a continuum of CD8 $+\mathrm{T}$ cell phenotypes. Immunity 36, 142-152 (2012).

35. Probert, C. S., Saubermann, L. J., Balk, S. \& Blumberg, R. S. Repertoire of the alpha beta T-cell receptor in the intestine. Immunol. Rev. 215, 215-225 (2007).

36. Regnault, A. et al. The expansion and selection of $\mathrm{T}$ cell receptor alpha beta intestinal intraepithelial T cell clones. Eur. J. Immunol. 26, 914-921 (1996).

37. Manfras, B. J. et al. Oligoclonal CD8 + T-cell expansion in patients with chronic hepatitis $\mathrm{C}$ is associated with liver pathology and poor response to interferon-alpha therapy. J. Clin. Immunol. 24, 258-271 (2004).

38. Bachelez, H. et al. Oligoclonal expansion of HIV-specific cytotoxic CD8 T lymphocytes in the skin of HIV-1-infected patients with cutaneous pseudolymphoma. J. Clin. Invest. 101, 2506-2516 (1998).

39. Manfras, B. J., Reuter, S., Wendland, T. \& Kern, P. Increased activation and oligoclonality of peripheral CD8 $(+) \mathrm{T}$ cells in the chronic human helminth infection alveolar echinococcosis. Infect. Immun. 70, 1168-1174 (2002).

40. Monteiro, J., Hingorani, R., Peroglizzi, R., Apatoff, B. \& Gregersen, P. K. Oligoclonality of CD8 $+\mathrm{T}$ cells in multiple sclerosis. Autoimmunity 23, 127-138 (1996)

41. Chang, J. C. et al. CD8 + T-cells in psoriatic lesions preferentially use T-cell receptors V beta 3 and/or V beta 13.1 genes. Ann. N. Y. Acad. Sci. 756, 370-381 (1995).

42. Cikota, B. M., Brankovic-Magic, M. V., Jovic, V. S., Radulovic, S. S. \& Magic, Z. M. Analysis of T-cell clonality pattern in tumor samples of breast cancer patients. Int. J. Biol. Markers 20, 177-183 (2005).

43. Derniame, S., Vignaud, J. M., Faure, G. C., Bene, M. C. \& Massin, F. Comparative T-cell oligoclonality in lung, tumor and lymph nodes in human non-small cell lung cancer. Oncol. Rep. 13, 509-515 (2005).

44. Caignard, A. et al. Evidence for T-cell clonal expansion in a patient with squamous cell carcinoma of the head and neck. Cancer Res. 54, 1292-1297 (1994).

45. Miconnet, I. Probing the T-cell receptor repertoire with deep sequencing. Curr. Opin. HIV AIDS 7, 64-70 (2012).

46. Young, M. H. et al. MAIT cell recognition of MR1 on bacterially infected and uninfected cells. PLoS ONE 8, e53789 (2013).
47. Meierovics, A., Yankelevich, W. J. \& Cowley, S. C. MAIT cells are critical for optimal mucosal immune responses during in vivo pulmonary bacterial infection. Proc. Natl Acad. Sci. USA 110, E3119-E3128 (2013).

48. Porcelli, S., Morita, C. T. \& Brenner, M. B. CD1b restricts the response of human CD4-8- T lymphocytes to a microbial antigen. Nature 360, 593-597 (1992).

49. De Libero, G. \& Lanzavecchia, A. Establishment of human double-positive thymocyte clones. J. Exp. Med. 170, 303-308 (1989).

50. Noppen, C. et al. C-type lectin-like receptors in peptide-specific HLA class I-restricted cytotoxic T lymphocytes: differential expression and modulation of effector functions in clones sharing identical TCR structure and epitope specificity. Eur. J. Immunol. 28, 1134-1142 (1998).

51. Robins, H. S. et al. Comprehensive assessment of T-cell receptor beta-chain diversity in alphabeta T cells. Blood 114, 4099-4107 (2009).

52. Carlson, C. S. et al. Using synthetic templates to design an unbiased multiplex PCR assay. Nat. Commun. 4, 2680 (2013).

53. Robins, H. et al. Ultra-sensitive detection of rare $\mathrm{T}$ cell clones. J. Immunol. Methods. 375, 14-19 (2012).

\section{Acknowledgements}

We thank Lin Jian'er and Lena Angman for TCR sequencing, Vincenza Carafa for tissue RNAs, SIgN flow cytometry platform for cell sorting and Immunomonitoring platform for Luminex experiments. We also thank Lucy Robinson of Insight Editing London for critical reading and editing of the manuscript, and Maria Romanova for art work. This work was supported by the core funds of the Department of Biomedicine, SIgN and by the Swiss National Fond Grant 3100AO-122464/1

\section{Author contributions}

M.L. designed the studies, performed the experiments and analysed the data. A.K., A.C., B.P., A.S., A.T. and L.Q. performed cellular and molecular experiments. B. L. and M.P. performed bioinformatics analyses. F.Z. contributed to deep-sequences experiments. E.N supervised Cytof analysis. P.S., A.B. and L.T. provided bacterial and tissue samples. L.M. and G.D.L. conceived the experiments and oversaw study design and data analysis. M.L. L.M. and G.D.L. wrote the manuscript. All authors discussed the results and commented on the manuscript.

\section{Additional information}

Accession codes: TCR $\beta$ deep-sequencing data (ImmunoSeEQ) has been deposited in the NCBI Sequence Read Archive under accession code SRP040254. Deep RNA-sequencing data set has been deposited in the Gene Expression Omnibus repository under accession code GSE56055

Supplementary Information accompanies this paper at http://www.nature.com/ naturecommunications

Competing financial interests: The authors declare no competing financial interests.

Reprints and permission information is available online at http://npg.nature.com/ reprintsandpermissions/

How to cite this article: Lepore, M. et al. Parallel T-cell cloning and deep sequencing of human MAIT cells reveal stable oligoclonal TCR $\beta$ repertoire. Nat. Commun. 5:3866 doi: $10.1038 /$ ncomms4866 (2014) 


\section{Corrigendum: Parallel T-cell cloning and deep sequencing of human MAIT cells reveal stable oligoclonal TCR $\beta$ repertoire}

Marco Lepore, Artem Kalinichenko, Alessia Colone, Bhairav Paleja, Amit Singhal, Andreas Tschumi, Bernett Lee, Michael Poidinger, Francesca Zolezzi, Luca Quagliata, Peter Sander, Evan Newell, Antonio Bertoletti, Luigi Terracciano, Gennaro De Libero \& Lucia Mori

Nature Communications 5:3866 doi: 10.1038/ncomms4866 (2014); Published 15 May 2014; Updated 11 Jul 2014

The original version of this Article contained a typographical error in the spelling of the author Artem Kalinichenko, which was incorrectly given as Artem Kalinicenko. This has now been corrected in both the PDF and HTML versions of the Article. 